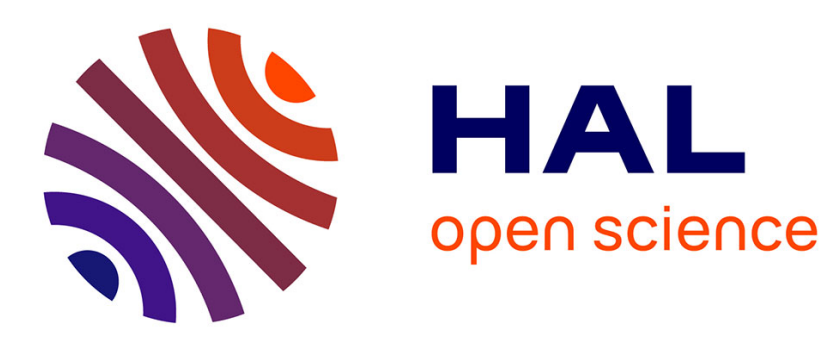

\title{
Hyperspectral unmixing with spectral variability using adaptive bundles and double sparsity
}

Tatsumi Uezato, Mathieu Fauvel, Nicolas Dobigeon

\section{To cite this version:}

Tatsumi Uezato, Mathieu Fauvel, Nicolas Dobigeon. Hyperspectral unmixing with spectral variability using adaptive bundles and double sparsity. 2020. hal-01797092

\section{HAL Id: hal-01797092 \\ https://hal.science/hal-01797092}

Preprint submitted on 21 Sep 2020

HAL is a multi-disciplinary open access archive for the deposit and dissemination of scientific research documents, whether they are published or not. The documents may come from teaching and research institutions in France or abroad, or from public or private research centers.
L'archive ouverte pluridisciplinaire HAL, est destinée au dépôt et à la diffusion de documents scientifiques de niveau recherche, publiés ou non, émanant des établissements d'enseignement et de recherche français ou étrangers, des laboratoires publics ou privés. 


\title{
Hyperspectral Unmixing With Spectral Variability Using Adaptive Bundles and Double Sparsity
}

\author{
Tatsumi Uezato $^{\circledR}$, Mathieu Fauvel, Senior Member, IEEE, and Nicolas Dobigeon ${ }^{\circledR}$, Senior Member, IEEE
}

\begin{abstract}
Spectral variability is one of the major issues when conducting hyperspectral unmixing. Within a given image composed of some elementary materials (herein referred to as endmember classes), the spectral signatures characterizing these classes may spatially vary due to intrinsic component fluctuations or external factors (illumination). These redundant multiple endmember spectra within each class adversely affect the performance of unmixing methods. This paper proposes a mixing model that explicitly incorporates a hierarchical structure of redundant multiple spectra representing each class. The proposed method is designed to promote sparsity on the selection of both spectra and classes within each pixel. The resulting unmixing algorithm is able to adaptively recover several bundles of endmember spectra associated with each class and robustly estimate abundances. In addition, its flexibility allows a variable number of classes to be present within each pixel of the hyperspectral image to be unmixed. The proposed method is compared with other state-ofthe-art unmixing methods that incorporate sparsity using both simulated and real hyperspectral data. The results show that the proposed method can successfully determine the variable number of classes present within each class and estimate the corresponding class abundances.
\end{abstract}

Index Terms - Endmember variability, hyperspectral imaging, sparse unmixing, spectral unmixing.

\section{INTRODUCTION}

$\mathbf{H}$ YPERSPECTRAL analysis has received an increasing attention because of its high spectral resolution, which enables a variety of objects to be identified and classified [1]. Mixed pixels caused by the presence of multiple objects within a single pixel adversely affect the performance of hyperspectral analysis [2]. To address this problem, a wide variety of spectral unmixing methods have been developed over the last decades [3]-[6]. Spectral unmixing methods aim at decomposing a mixed spectrum into a collection of reference spectra (known as endmembers) characterizing the macroscopic materials present in the scene and their respective

Manuscript received April 27, 2018; revised October 2, 2018; accepted December 16, 2018. This work was supported in part by EU FP7 through the ERANETMED JC-WATER Program, in part by MapInvPlnt Project under Grant ANR-15-NMED-0002-02, and in part by the MUESLI IDEX ATS Project, Toulouse INP. (Corresponding author: Tatsumi Uezato.)

$\mathrm{T}$. Uezato and N. Dobigeon are with the University of Toulouse, IRIT/INP-ENSEEIHT, CNRS, 31071 Toulouse, France (e-mail: tatsumi.uezato@ riken.jp; nicolas.dobigeon@enseeiht.fr).

M. Fauvel is with the Centre d'Études Spatiales de la BIOsphere, Universitê de Toulouse, CNES/CNRS/IRD/UPS/INRA, 31401 Toulouse, France (e-mail: mathieu.fauvel@inra.fr).

Color versions of one or more of the figures in this paper are available online at http://ieeexplore.ieee.org.

Digital Object Identifier 10.1109/TGRS.2018.2889256 proportions (known as abundances) in each image pixel [2]. Despite the large number of developed spectral unmixing methods, there are still major challenges for accurate estimates of endmember signatures and abundances [3]. Among these challenges, endmember variability may lead to large amounts of errors in abundance estimates [7]. It results from the fact that each endmember can rarely be represented by a unique spectral signature. Conversely, it is subject to so-called spectral variability, e.g., caused by variations in the acquisition process, the intensity of illumination, or other physical characteristics of the materials [8], [9]. Taking this endmember variability into account during the spectral unmixing process is one of the keys for successful application of spectral unmixing [10].

The methods that incorporate endmember variability can be categorized into two main approaches (see [7], [10], [11] for recent overviews). The first approach relies on the definition of a set of multiple spectral signatures, referred to as endmember bundles, to characterize each endmember class. Endmember bundles can be collected from field campaign or can be extracted from data itself using endmember bundle extraction methods [9], [12], [13]. The advantage of this approach is the simple representation of the endmember variability neither assuming any particular distribution nor adding additional terms describing this variability. This can be achieved by simply selecting physically possible spectral signatures from prior information (i.e., endmember bundles). Endmember bundles can be validated by experts in order to provide accurate representation about endmember variability [12]. Although traditional methods incorporating endmember bundles (see [14]) are known to be computationally expensive, more efficient methods have been recently developed and have shown great potential [15]-[18]. However, as pointed out in [19], it is unlikely that the endmember bundles completely represent endmember variability present in an image. Such incomplete endmember bundles may lead to poor estimates of abundances. The second approach uses physical or statistical descriptions of the endmember variability. More precisely, these methods describe the endmember variability thanks to a statistical distribution [20] or by incorporating additional variability terms in the mixing model [21]-[23]. This approach can potentially incorporate endmember bundles as supervised methods (see [24], [25]). The advantage of this approach results from the adaptive learning of the endmember variability. Indeed, state-of-the-art methods such as those recently introduced in [21]-[23] enable endmember spectra to spatially vary within each pixel in order to describe 
endmember variability. This is important since the endmember spectra to be used for the abundance estimation can be different between pixels. However, estimating endmember variability is a challenging task, especially when large amounts of endmember variability are present in an image. In addition, the statistical distribution or additional terms used in these methods may be overly simplified to represent endmember variability.

Both approaches demonstrate benefits and drawbacks. A natural question arises: is it possible to combine the strong advantages of both approaches to robustly represent endmember variability? This paper addresses the question and introduces a novel spectral unmixing method that bridges the gap between the aforementioned two approaches with the help of a double sparsity-based method inspired by Rubinstein et al. [26]. Specifically, the proposed method aims at adaptively recovering endmember spectra within each pixel to describe endmember variability while preserving simple representation. The proposed method is closely related to the existing methods. Thus, the main contributions of this paper are threefold: 1) to propose a novel spectral unmixing method that incorporates adaptive endmember bundles through double sparsity representation; 2) to give a systematic review of related work and show the relationship between the proposed method and existing methods; and 3) to provide comparison between the proposed method and other sparsity-based methods.

This paper is organized as follows. Section II describes related works and existing methods, while highlighting their inherent drawbacks. In Section III, a novel mixing model that incorporates endmember variability is proposed and its relationships with existing methods are discussed. Section IV introduces an associated unmixing algorithm designed to recover the endmember classes, adaptive bundles, and abundances. Sections V and VI show experimental results obtained from simulated data and real hyperspectral images. Finally, the conclusion is drawn in Section VII.

\section{Related Works AND Issues RAISED BY EXISTING METHODS}

\section{A. Conventional (Variability-Free) Linear Mixing Model}

Let $\mathbf{y}_{i} \in \mathbb{R}^{L \times 1}$ denote the $L$-spectrum measured at the $i$ th pixel of a hyperspectral image. According to the linear mixing model (LMM), the observed spectrum of the $i$ th pixel $\mathbf{y}_{i}$ is approximated by a weighted linear combination of endmember spectra and abundance fractions

$$
\mathbf{y}_{i}=\mathbf{M a}_{i}+\mathbf{n}_{i}
$$

where $\mathbf{M} \in \mathbb{R}^{L \times K}$ is the matrix of the spectral signatures associated with the $K$ endmember classes, $\mathbf{a}_{i}=\left[a_{1 i}, \ldots, a_{K i}\right]^{\mathrm{T}} \in$ $\mathbb{R}^{K \times 1}$ is the abundance fractions of the pixel, and $\mathbf{n}_{i} \in$ $\mathbb{R}^{L \times 1}$ represents noise and modeling error. LMM is generally accompanied by abundance nonnegativity constraint (ANC) and the abundance sum-to-one constraint (ASC)

$$
\forall k, \forall i, \quad a_{k i} \geq 0, \quad \text { and } \forall i, \quad \sum_{k=1}^{K} a_{k i}=1
$$

where $a_{k i}$ is the abundance fraction of the $k$ th class in the $i$ th pixel. This model implicitly relies on the following assumption: each endmember class in $\mathbf{M}$ is described by a unique spectrum and commonly used to unmix all the pixels of a given image. In other words, LMM does not account for spectral variability. However, as discussed previously, this is likely unrealistic because spectral variability is naturally observed in hyperspectral images, e.g., because of variations in illumination or physical intrinsic characteristics of materials [19].

\section{B. Linear Mixing Models Incorporating Endmember Bundles}

LMMs that consider endmember bundles have been developed. Endmember bundles can be extracted manually using the method developed in [12] or the ENVI software as done in [19]. Several methods that automatically extract endmember bundles have also been developed [9], [13], [27]. The automated endmember bundle extraction method proposed in [13] sequentially generates a number of subsets representing randomly selected pixels and extracts endmembers from each subset. Then, bundles are generated by grouping the extracted multiple endmembers. More recently, endmember bundle extraction methods that consider both spatial and spectral information have been developed [9], [27]. Endmember bundles can be potentially estimated by LMMs incorporating spectral variability [21]-[23]. These methods explicitly add a term representing endmember variability and, thus, estimate variable endmember spectra within each class. The estimated endmember spectra of each class can be subsequently grouped into endmember bundles. Although endmember bundles can be extracted by the above methods, in this paper, we assume that endmember bundles are a priori available and have been previously identified. This prevents unmixing results to be affected by the performance of the endmember bundle extraction step and enables fair comparison.

Multiple endmember spectral mixture analysis (MESMA) [14] allows the variability of the endmember spectrum representative of each class and a varying number of endmember classes present within each pixel. Although MESMA has been widely used for a variety of applications [14], [28], it owns several major limitations.

1) It is highly computationally expensive because MESMA needs to test a large number of combinations of endmember spectra [29].

2) MESMA tends to select an overestimated number of endmember classes because it uses the reconstruction error to select the appropriate combination of endmember spectra [30].

3) The performance of MESMA may significantly decrease when endmember spectra (or bundles) within each class do not completely represent the spectral variability [19].

To overcome these limitations, recent works have proposed a new class of methods that incorporate all endmember bundles defined as [15], [16]

$$
\mathbf{E}=\left[\mathbf{E}_{1}\left|\mathbf{E}_{2}\right| \cdots \mid \mathbf{E}_{K}\right]
$$

where $\mathbf{E}_{k} \in \mathbb{R}^{L \times N_{k}}$ represents a set of endmember spectra (i.e., bundle) characterizing the $k$ th class, $N_{k}$ is the number of 
endmember spectra in the $k$ th class, and $N$ is the total number of endmember spectra of all classes with $N=\sum_{k=1}^{K} N_{k}$. Generalizing LMM in (1), those methods first model a given observed pixel spectrum with respect to (w.r.t.) all spectra in endmember bundles and corresponding multiple abundances

$$
\mathbf{y}_{i}=\mathbf{E r}_{i}+\mathbf{n}_{i}
$$

where $\mathbf{r}_{i} \in \mathbb{R}^{N \times 1}$ is multiple abundance fractions corresponding to each spectrum of the endmember bundles $\mathbf{E}$. As for LMM, ASC or ANC can also be imposed to $\mathbf{r}_{i}$. As a second step, multiple abundance fractions $\mathbf{r}_{i}$ are summed within each class to generate a single abundance fraction for each class

$$
\mathbf{a}_{i}=\mathbf{G}^{T} \mathbf{r}_{i}
$$

with

$$
\mathbf{G}=\left[\begin{array}{cccc}
\mathbf{1}_{N_{1}} & \mathbf{0}_{N_{1}} & \cdots & \mathbf{0}_{N_{1}} \\
\mathbf{0}_{N_{2}} & \mathbf{1}_{N_{2}} & \cdots & \mathbf{0}_{N_{2}} \\
\vdots & \vdots & \ddots & \vdots \\
\mathbf{0}_{N_{K}} & \mathbf{0}_{N_{K}} & \cdots & \mathbf{1}_{N_{K}}
\end{array}\right]
$$

where $\mathbf{1}_{N_{k}} \in \mathbb{R}^{N_{k} \times 1}$ is a column vector of ones and $\mathbf{0}_{N_{k}} \in$ $\mathbb{R}^{N_{k} \times 1}$ represent an $N_{k}$-dimensional vector whose components are zeros. While these two steps are conducted separately in [15] and [16], they can also be considered jointly within a multitask Gaussian process framework [17], [19]. Even if these methods have been shown to be effective, a large number of endmember spectra within each class may be redundant. In such case, following a model selection inspiration, Veganzones et al. [15] introduce a complementary sparsity regularization on the multiple abundance vectors

$$
\begin{aligned}
& \min _{\mathbf{r}_{i}} \frac{1}{2}\left\|\mathbf{E} \mathbf{r}_{i}-\mathbf{y}_{i}\right\|_{2}^{2}+\lambda_{r}\left\|\mathbf{r}_{i}\right\|_{1} \\
& \text { s.t. } \forall i, \quad \mathbf{r}_{i} \succeq
\end{aligned}
$$

where $\succeq$ represents the element-wise comparison, $\|\cdot\|_{2}$ is the $\ell_{2}$-norm, and $\|\cdot\|_{1}$ is the $\ell_{1}$-norm which is known to promote sparsity. Once the multiple abundance vector $\mathbf{r}_{i}$ has been estimated, it is normalized in order to reduce the effects of multiplicative factors and satisfy ASC. Following the same approach, further sparsity can be imposed using $\ell_{p}$-norm [31] or reweighted $\ell_{1}$-approaches [32], [33]. Overall, this sparsity property allows the selection of a smaller number of endmember spectra. However, it may not lead to the selection of a smaller number of endmember classes. Conversely, to promote sparsity on the number of endmember classes, one strategy consists in formulating the unmixing problem through a sparse group lasso [34]

$$
\begin{aligned}
& \min _{\mathbf{r}_{i}}\left\{\frac{1}{2}\left\|\sum_{k=1}^{K} \mathbf{E}_{k}\left(\mathbf{g}_{k} \odot \mathbf{r}_{i}\right)-\mathbf{y}_{i}\right\|_{2}^{2}\right. \\
& \left.\quad+\lambda_{g} \sum_{k=1}^{K}\left\|\mathbf{g}_{k} \odot \mathbf{r}_{i}\right\|_{2}+\lambda_{r}\left\|\mathbf{r}_{i}\right\|_{1}\right\} \\
& \text { s.t. } \quad \forall i, \quad \mathbf{r}_{i} \succeq 0
\end{aligned}
$$

where $\mathbf{g}_{k}$ is the $k$ th column of $\mathbf{G}, \odot$ is the element-wise product, and thus, $\mathbf{g}_{k} \odot \mathbf{r}_{i}$ extracts the elements in $\mathbf{r}_{i}$ belonging to the $k$ th class. This approach has the great advantage of promoting sparsity in both the number of endmember spectra and the number of endmember classes. Another strategy relies on the concept of "social sparsity" that can exploit the structure of endmember bundles more explicitly [18]. The method assumes that $\mathbf{r}_{i}$ can be partitioned into $K$ groups representing each endmember class, leading to the optimization problem

$$
\begin{aligned}
& \min _{\mathbf{r}_{i}}\left\{\frac{1}{2}\left\|\mathbf{E r}_{i}-\mathbf{y}_{i}\right\|_{2}^{2}+\lambda_{r}\left(\sum_{k=1}^{K}\left\|\mathbf{g}_{k} \odot \mathbf{r}_{i}\right\|_{p}^{q}\right)^{\frac{1}{q}}\right\} \\
& \text { s.t. } \forall i, \quad \forall n, \quad \mathbf{r}_{i} \succeq 0, \quad \sum_{n=1}^{N} r_{n i}=1
\end{aligned}
$$

where $\|\cdot\|_{p}$ is the $\ell_{p}$-norm and $r_{n i}$ is the $n$th multiple abundance fraction of the $i$ th pixel. Finally, abundances associated each endmember class can be obtained by summing the multiple abundances within each class as in (5). This method can be considered as a generalized model, since by adjusting the values of $(p, q)$, it boils down to the group lasso $(2,1)$, the elitist lasso $(1,2)$, or the fractional case $(1, q)$ where $q \in \mathbb{N}$ and $0<q<1$ [18]. The fractional case promotes sparsity of the selection of both endmember classes and spectra within each class. The aforementioned methods that incorporate all endmember bundles can potentially recover pixel-wise endmember variability, at the price of a thorough postprocessing analysis. More precisely, the pixel-wise endmember variability can be estimated by a weighted combination of all possible endmember spectra within each class, e.g., as done in [35].

All of the aforementioned sparsity-based methods still suffer from the following limitations.

1) Interpretation of the Models: The models are designed to estimate multiple abundance fractions $\mathbf{r}$ corresponding to each spectrum in endmember bundles. Although the models can implicitly consider abundances for each class or endmember spectra for each pixel, they cannot independently describe pixel-wise endmember spectra. In order to generate pixel-wise endmember spectra, a thorough postprocessing analysis is required using a normalization technique [35].

2) Adaptability to Describe Endmember Variability: ASC imposed on $\mathbf{r}_{i}$ does not allow a consistent description of the endmembers within each pixel. In addition, it cannot explicitly capture adaptive and hierarchical structure of endmember spectra for each pixel.

To overcome these two shortcomings, this paper capitalizes on this abundant literature to design a new multiple endember mixing model introduced in Section III.

\section{A. MEMM}

\section{Multiple Endmember Mixing Models}

The proposed model relies on three main ingredients, namely, endmember bundles, bundling coefficients, and abundances. According to this model, each endmember bundle is mixed to provide a suitable and adaptive endmember spectrum used to unmix a given pixel. The proposed multiple endmember mixing model (MEMM) is defined as

$$
\mathbf{y}_{i}=\mathbf{E} \mathbf{B}_{i} \mathbf{a}_{i}+\mathbf{n}_{i}
$$


where $\mathbf{B}_{i} \in \mathbb{R}^{N \times K}$ gathers so-called bundling coefficients of the $i$ th pixel which decompose the endmember signatures according to the endmember bundles for the considered pixel. To enforce the bundle structure, the bundling coefficients $\mathbf{B}_{i}$ associated with the pixel is defined as the following block-diagonal matrix:

$$
\mathbf{B}_{i}=\left[\begin{array}{cccc}
\mathbf{b}_{1 i} & \mathbf{0}_{N_{1}} & \cdots & \mathbf{0}_{N_{1}} \\
\mathbf{0}_{N_{2}} & \mathbf{b}_{2 i} & \cdots & \mathbf{0}_{N_{2}} \\
\vdots & \vdots & \ddots & \vdots \\
\mathbf{0}_{N_{K}} & \mathbf{0}_{N_{K}} & \cdots & \mathbf{b}_{K i}
\end{array}\right]
$$

where $\mathbf{b}_{k i} \in \mathbb{R}^{N_{k} \times 1}$ is the bundling coefficients for the $k$ th class at the $i$ th pixel. Each bundling coefficient must be nonnegative, and the bundling vector $\mathbf{b}_{k i}$ is expected to be sparse. Indeed, multiple endmember spectra within each class are usually redundant and only a few endmember spectra within each class should be enough to unmix a pixel. This property can be induced by considering the following bundling constraints:

$$
\forall i, \mathbf{B}_{i} \succeq 0 \text { and }\left\|\mathbf{B}_{i}\right\|_{0}=\sum_{k=1}^{K}\left\|\mathbf{b}_{k i}\right\|_{0} \leq s
$$

where $\|\cdot\|_{0}$ is the $\ell_{0}$-norm that counts the number of nonzero elements and $s$ is the maximum number of nonzero elements in $\mathbf{B}_{i}$, i.e., the maximum number of endmembers to be used within each class to describe the pixel. The ANC and the ASC are usually imposed. In addition, in this paper, complementary sparsity is imposed on each abundance vector, i.e.,

$$
\forall k, \forall i, a_{k i} \geq 0, \quad \text { and } \forall i, \quad \sum_{k=1}^{K} a_{k i}=1, \quad\left\|\mathbf{a}_{i}\right\|_{0} \leq v
$$

where $v$ is the number of endmember classes to be used to decompose the image pixel.

\section{B. $M E M M s$}

The sparsity constraint (12) applied to $\mathbf{B}$ can be slightly modified to obtain another meaningful set of constraints

$$
\forall i, \mathbf{B}_{i} \succeq 0 \text { and } \forall k, \forall i,\left\|\mathbf{b}_{k i}\right\|_{0} \leq 1 .
$$

The resulting model, referred to as $\mathrm{MEMM}_{s}$ in what follows, is designed to generate at most one scaled endmember spectrum for each class. Details on the optimization of $\mathrm{MEMM}_{s}$ are available in [36].

\section{Relationships Between MEMM and Existing Models}

1) $M E M M_{s}$ and MESMA: When the sparsity constraint on the abundances $\left\|\mathbf{a}_{i}\right\|_{0} \leq v$ in (13) is not considered, the optimization problem associated with the $\mathrm{MEMM}_{s}$ model described in Section III-B is equivalent to MESMA and sparse MESMA [37]. Unlike MESMA that considers the reconstruction error to determine the optimal combination of endmember classes within each pixel, $\mathrm{MEMM}_{s}$ incorporates the sparsity constraint to select the optimal combination. This prevents a larger number of endmember classes to be selected for each pixel.
2) MEMM and Pixel-Wise Endmember Variability Models: By denoting $\tilde{\mathbf{M}}_{i}=\mathbf{E B}_{i}$, the equivalent endmember matrix associated with the $i$ th pixel, MEMM models the observed pixel spectra as

$$
\mathbf{y}_{i}=\tilde{\mathbf{M}}_{i} \mathbf{a}_{i}+\mathbf{n}_{i}
$$

where $\tilde{\mathbf{M}}_{i}$ can be interpreted as a set of $K$ spatially varying endmember spectra. This approach has also been adopted in recent works to incorporate endmember variability as additive factors [22], multiplicative factors [21], [38], or a combination of additive and multiplicative factors [23]. In particular, when $N_{1}=\ldots, N_{k}=1$ in (3), the endmember bundles $\mathbf{E}_{1}, \ldots, \mathbf{E}_{K}$ are reduced to unique endmember spectra characterizing each class. The associated bundling coefficient matrix $\mathbf{B}=\operatorname{diag}\left[b_{1}, \ldots, b_{K}\right]$ is diagonal, where each coefficient $b_{k}$ scales the corresponding endmember spectrum $\mathbf{E}_{k}(k=1, \ldots, K)$. Thus, MEMM generalizes the recently introduced extended LMM [21].

However, MEMM is different from the aforementioned methods since it resorts to a priori information (i.e., endmember bundles) to model the endmember variability. More precisely, MEMM describes the admissible variability within an endmember class as the convex cone spanned by the corresponding bundles. As a consequence, per se, MEMM offers an adaptive description of the spectral variability even when predefined endmember bundles do not completely capture this variability within each class.

Finally, when the existing methods impose ASC onto $\mathbf{a}_{i}$, they assume that mixed spectra belong to the simplex spanned by the endmember bundles. In such case, pixel-wise endmember spectra can be generated as convex combinations of spectra within each bundle where the weight of each bundling coefficient corresponds to a normalized abundance of a spectrum in the bundle. However, this is a limited assumption since observed spectra may be outside the simplex, e.g., when affected by variations in illumination. MEMM imposes ASC only onto the abundances $\mathbf{a}_{i}$ of endmember classes and enables the bundling coefficients $\mathbf{B}_{i}$ to scale the endmember signature, e.g., to capture variability induced by varying illumination (see experiments in Section VII).

3) MEMM and Sparsity-Based Unmixing Methods: By setting $\mathbf{r}_{i}=\mathbf{B}_{i} \mathbf{a}_{i}$, MEMM in (10) can be rewritten as

$$
\mathbf{y}_{i}=\mathbf{E r}_{i}+\mathbf{n}_{i}
$$

similar to the existing models discussed in Section II. The main difference is that MEMM enables the multiple abundances $\mathbf{r}_{i}$ to be decomposed into bundling coefficients $\mathbf{B}_{i}$ within each class and abundances $\mathbf{a}_{i}$, resulting into a bilayer description of the abundances. This hierarchical decomposition has been also adopted by unmixing methods based on multilayer nonnegative matrix factorization [39]. However, each layer induced by MEMM (i.e., bundling matrix and abundance vector) has a clear and meaningful role. In addition, MEMM complements this bilayer hierarchy with a twofold structured, physically-motivated sparsity imposed on the multiple abundance vector $\mathbf{r}_{i}$. This bilevel sparsity has the significant advantage of reducing overfitting, and one may expect a 
significant improvement of stability and interpretability of the abundance estimates.

The intrinsic structure of MEMM is also similar to the recently developed methods based on robust constrained matrix factorization [40] and kernel archetypoid analysis [41]. These methods model a set $\mathbf{Y}=\left[\mathbf{y}_{1}, \ldots, \mathbf{y}_{P}\right] \in \mathbb{R}^{L \times P}$ of $P$ pixel spectra as

$$
\mathbf{Y}=\mathbf{Y C A}+\mathbf{N}
$$

where $\mathbf{C} \in \mathbb{R}^{P \times K}$ is a matrix gathering a set of coefficients, $\mathbf{A}=\left[\mathbf{a}_{1}, \ldots, \mathbf{a}_{P}\right] \in \mathbb{R}^{K \times P}$ is the abundance matrix and $\mathbf{N}=\left[\mathbf{n}_{1}, \ldots, \mathbf{n}_{P}\right] \in \mathbb{R}^{L \times N}$ is the error and noise matrix. Sparsity (induced by ASC or the use of $\ell_{0}$-pseudonorm) and nonnegativity constraints are imposed onto each column of $\mathbf{C}$ and $\mathbf{Y C}$ can be interpreted as synthetic endmember spectra. These methods use the subset of whole image pixels to generate synthetic endmember spectra that are fixed within each image. On the other hand, MEMM uses the subset of endmember bundles to generate synthetic endmember spectra that may be different for each pixel.

Finally, MEMM is closely related to the fractional lasso-based method that has been recently proposed in [35]. Both methods impose sparsity for the selection of both endmember classes and endmember spectra, in a similar manner proposed in [34]. In addition, they can impose ASC and also recover pixel-wise endmember spectra and abundances for each endmember class. However, there are differences between these two methods.

1) MEMM defines bundling coefficients and, thus, can explicitly express pixel-wise endmember spectra $\tilde{\mathbf{M}}_{i}$ and abundances $\mathbf{a}_{i}$ for each endmember class in the unmixing model. On the other hand, the method proposed in [35] is designed to estimate multiple abundance fractions $\mathbf{r}_{i}$ for each possible endmember spectrum among endmember bundles in the unmixing model. Abundances for each endmember class or pixel-wise endmember spectra are subsequently recovered by conducting postprocessing steps. In other words, MEMM estimates the bundling coefficients independently whereas in [35], they are computed from the multiple abundance fractions.

2) While the bundling coefficients are only required to be positive in MEMM, both positivity and sum-to-one constraints are imposed by the model introduced in [35]. This leads to a different geometrical interpretation of pixel-wise endmember spectra (see Fig. 1). Pixel-wise endmember spectra derived by MEMM are inside the conic hull defined by each endmember bundle. The conic hull can also be interpreted as scaling factors discussed in [15] and [21] which are important, e.g., to describe varying illumination. On the other hand, the method in [35] generates pixel-wise endmember spectra as the convex combination of endmember spectra within each endmember bundle. Note that, if MEMM considers a sum-to-one constraint for each column in B, MEMM can also consider the convex hull described by each endmember bundle.

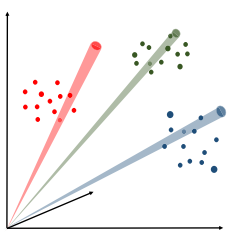

(a)

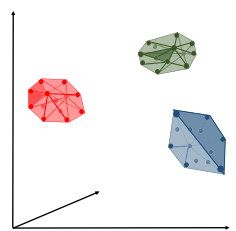

(b)

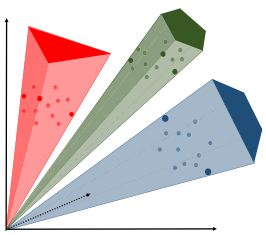

(c)
Fig. 1. Geometrical interpretation of potential endmember variability derived by different methods. Different colors represent different endmember classes. Points represent given endmember spectra within each class. Transparent colors represent potential endmember variability generated by the methods. (a) Potential endmember variability described by a line using $\mathrm{MEMM}_{s}$ or MESMA. (b) Potential endmember variability described by convex hull using the fractional lasso-based method or other methods that imposes ASC on $\mathbf{r}$ (see [16]). (c) Potential endmember variability described by convex cone using the proposed MEMM.

\section{MEMM-BASEd UnMiXing ALgORITHM}

Unmixing according to the proposed MEMM can be formulated as the minimization problem

$$
\begin{aligned}
& \min _{\mathbf{B}_{i}, \mathbf{a}_{i}} \frac{1}{2}\left\|\mathbf{E} \mathbf{B}_{i} \mathbf{a}_{i}-\mathbf{y}_{i}\right\|_{2}^{2} \\
& \text { s.t. } \forall k, \forall i, \quad a_{k i} \geq 0, \quad \sum_{k=1}^{K} a_{k i}=1, \quad\left\|\mathbf{a}_{i}\right\|_{0} \leq v, \\
& \qquad \mathbf{B}_{i} \succeq 0, \quad\left\|\mathbf{B}_{i}\right\|_{0} \leq s .
\end{aligned}
$$

This minimization problem is similar to the double sparsity-inducing method proposed in [26]. Using an alternative formulation, the minimization problem can be written as the following nonconvex minimization problem:

$$
\min _{\mathbf{B}_{i}, \mathbf{a}_{i}} \mathcal{J}\left(\mathbf{B}_{i}, \mathbf{a}_{i}\right)=\left\{f\left(\mathbf{B}_{i}, \mathbf{a}_{i}\right)+h\left(\mathbf{B}_{i}\right)+g\left(\mathbf{a}_{i}\right)\right\}
$$

with

$$
\begin{aligned}
f\left(\mathbf{B}_{i}, \mathbf{a}_{i}\right) & =\frac{1}{2}\left\|\mathbf{E} \mathbf{B}_{i} \mathbf{a}_{i}-\mathbf{y}_{i}\right\|_{2}^{2} \\
h\left(\mathbf{B}_{i}\right) & =\iota_{\mathbb{R}_{+}}\left(\mathbf{B}_{i}\right)+\lambda_{b}\left\|\mathbf{B}_{i}\right\|_{0} \\
g\left(\mathbf{a}_{i}\right) & =\iota_{\mathbb{S}}\left(\mathbf{a}_{i}\right)+\lambda_{a}\left\|\mathbf{a}_{i}\right\|_{0}
\end{aligned}
$$

where $\lambda_{a}$ and $\lambda_{b}$ are parameters which control the balance between the data fitting term and the sparse regularizations, $\iota_{\mathcal{C}}(\mathbf{x})$ is the indicator function on the set $C$ [i.e., $\iota_{\mathcal{C}}(\mathbf{x})=0$ when $\mathbf{x} \in \mathcal{C}$ whereas $\iota_{\mathcal{C}}(\mathbf{x})=\infty$ when $\left.\mathbf{x} \notin \mathcal{C}\right]$, and $\mathbb{S}$ is the simplex defined by the ASC and ANC. Promoting the sparsity of the solution through penalized regularizations rather than a hard constraint allows the contribution of the sparsity term to be adjusted w.r.t. the data-fitting term rather than directly constraining the maximum number of classes. This flexibility allows different numbers of selected endmember spectra or classes to be selected for each pixel. Solving this optimization problem is challenging since the regularization functions $h$ and $g$ are nonconvex and nonsmooth. However, it can be tackled thanks to the proximal alternating linearized minimization (PALM) [42]. With guarantees to converge to a critical point, PALM iteratively updates the parameters $\mathbf{a}_{i}$ and $\mathbf{B}_{i}$ by alternatively minimizing the objective function w.r.t. these 


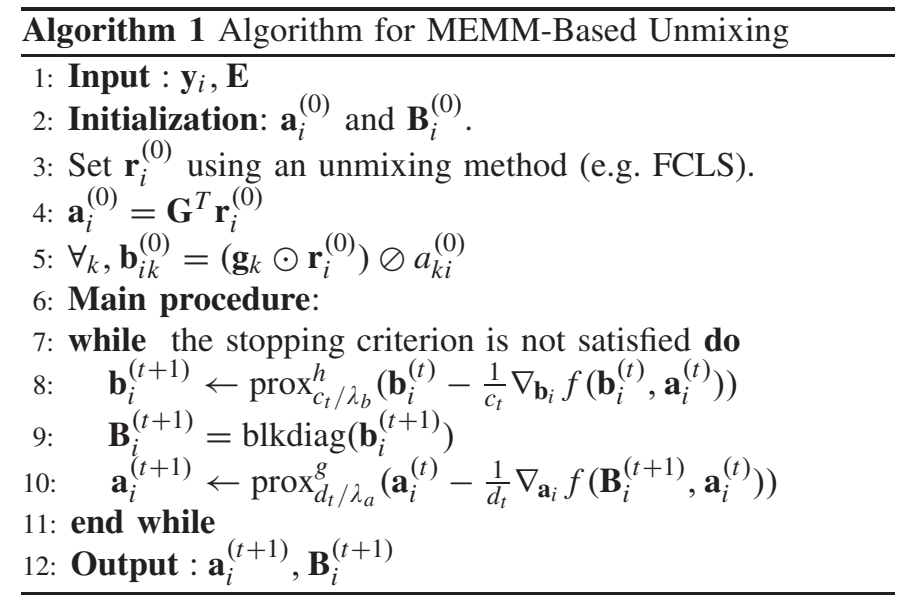

parameters, i.e., by solving the following proximal problems:

$$
\begin{aligned}
& \mathbf{B}_{i}^{(t+1)} \in \min _{\mathbf{B}_{i}}\{ h\left(\mathbf{B}_{i}\right)+\left\langle\mathbf{B}_{i}-\mathbf{B}_{i}^{(t)}, \nabla_{\mathbf{B}_{i}} f\left(\mathbf{B}_{i}^{(t)}, \mathbf{a}_{i}^{(t)}\right)\right\rangle \\
&+\left.\frac{c_{t}}{2}\left\|\mathbf{B}_{i}-\mathbf{B}_{i}^{(t)}\right\|_{2}^{2}\right\} \\
& \mathbf{a}_{i}^{(t+1)} \in \min _{\mathbf{a}_{i}}\left\{\begin{array}{l}
g\left(\mathbf{a}_{i}\right)+\left\langle\mathbf{a}_{i}-\mathbf{a}_{i}^{(t)}, \nabla_{\mathbf{a}_{i}} f\left(\mathbf{B}_{i}^{(t+1)}, \mathbf{a}_{i}^{(t)}\right)\right\rangle \\
\left.+\frac{d_{t}}{2}\left\|\mathbf{a}_{i}-\mathbf{a}_{i}^{(t)}\right\|_{2}^{2}\right\} .
\end{array}\right.
\end{aligned}
$$

The pseudocode for MEMM is shown in Algorithm 1, and these two steps are described in what follows.

\section{A. Optimization w.r.t. $\mathbf{B}_{i}$}

To optimize only w.r.t. the diagonal entries in $\mathbf{B}_{i}$, the objective function can be rewritten with the following decomposition:

$$
\begin{aligned}
f\left(\mathbf{b}_{i}, \mathbf{a}_{i}\right) & =\frac{1}{2}\left\|\mathbf{U}_{i} \mathbf{b}_{i}-\mathbf{y}_{i}\right\|_{2}^{2} \\
h\left(\mathbf{b}_{i}\right) & =\imath_{\mathbb{R}_{+}}\left(\mathbf{b}_{i}\right)+\lambda_{b}\left\|\mathbf{b}_{i}\right\|_{0}
\end{aligned}
$$

where

$$
\begin{aligned}
\mathbf{U}_{i} & =\left[\mathbf{E}_{1} a_{1 i}|\cdots| \mathbf{E}_{K} a_{K i}\right] \\
\mathbf{b}_{i} & =\left[\mathbf{b}_{1 i}^{T}, \mathbf{b}_{2 i}^{T}, \cdots, \mathbf{b}_{K i}^{T}\right]^{\mathrm{T}} .
\end{aligned}
$$

This leads to the following updating rule:

$$
\min _{\mathbf{b}_{i}}\left\{h\left(\mathbf{b}_{i}\right)+\frac{c_{t}}{2}\left\|\mathbf{b}_{i}-\left(\mathbf{b}_{i}^{(t)}-\frac{1}{c_{t}} \nabla_{\mathbf{b}} f\left(\mathbf{b}_{i}^{(t)}, \mathbf{a}_{i}^{(t)}\right)\right)\right\|_{2}^{2}\right\}
$$

where $\nabla_{\mathbf{b}_{i}} f\left(\mathbf{b}_{i}^{(t)}, \mathbf{a}_{i}^{(t)}\right)=\mathbf{U}_{i}^{T}\left(\mathbf{U}_{i} \mathbf{b}_{i}-\mathbf{y}_{i}\right)$. Using similar computations as in [42], this can be conducted as

$$
\mathbf{b}_{i}^{(t+1)} \in \operatorname{prox}_{c_{t} / \lambda_{b}}^{h}\left(\mathbf{b}_{i}^{(t)}-\frac{1}{c_{t}} \nabla_{\mathbf{b}_{i}} f\left(\mathbf{b}_{i}^{(t)}, \mathbf{a}_{i}^{(t)}\right)\right)
$$

where $c_{t}=\gamma_{m}\left\|\mathbf{U}_{i}^{T} \mathbf{U}_{i}\right\|_{\mathrm{F}}$ represents a step size for each iteration. The proximal operator associated with $f$ can be computed using the approach [42]. Finally, the bundling matrix $\mathbf{B}_{i}$ can be reconstructed as $\mathbf{B}_{i}=\operatorname{blkdiag}\left(\mathbf{b}_{i}\right)$, where blkdiag $(\cdot)$ generates the block diagonal matrix $\mathbf{B}_{i}$ from the vector $\mathbf{b}_{i}$.

\section{B. Optimization With Respect to $\mathbf{a}_{i}$}

To optimize w.r.t. $\mathbf{a}_{i}$, the objective function can be rewritten using the decomposition

$$
\begin{aligned}
f\left(\mathbf{B}_{i}, \mathbf{a}_{i}\right) & =\frac{1}{2}\left\|\tilde{\mathbf{M}}_{i} \mathbf{a}_{i}-\mathbf{y}_{i}\right\|_{2}^{2} \\
g\left(\mathbf{a}_{i}\right) & =\iota_{\mathbb{S}}\left(\mathbf{a}_{i}\right)+\lambda_{a}\left\|\mathbf{a}_{i}\right\|_{0}
\end{aligned}
$$

where $\tilde{\mathbf{M}}_{i}=\mathbf{E B}_{i}$. Thus, updating the abundance vector can be formulated as

$$
\min _{\mathbf{a}_{i}}\left\{g\left(\mathbf{a}_{i}\right)+\frac{d_{t}}{2}\left\|\mathbf{a}_{i}-\left(\mathbf{a}_{i}^{(t)}-\frac{1}{d_{t}} \nabla_{\mathbf{a}_{i}} f\left(\mathbf{B}_{i}^{(t+1)}, \mathbf{a}_{i}^{(t)}\right)\right)\right\|^{2}\right\}
$$

where $\nabla_{\mathbf{a}_{i}} f\left(\mathbf{B}_{i}^{(t+1)}, \mathbf{a}_{i}^{(t)}\right)=\tilde{\mathbf{M}}_{i}^{T}\left(\tilde{\mathbf{M}}_{i} \mathbf{a}_{i}-\mathbf{y}_{i}\right)$. Using the proximal operator, this can be written as

$$
\mathbf{a}_{i}^{(t+1)} \in \operatorname{prox}_{d_{t} / \lambda_{a}}^{g}\left(\mathbf{a}_{i}^{(t)}-\frac{1}{d_{t}} \nabla_{\mathbf{a}_{i}} f\left(\mathbf{B}_{i}^{(t+1)}, \mathbf{a}_{i}^{(t)}\right)\right)
$$

where $d_{t}=\gamma_{a}\left\|\tilde{\mathbf{M}}_{i}^{T} \tilde{\mathbf{M}}_{i}\right\|_{\mathrm{F}}$ represents a step size for each iteration. Moreover the proximal mapping associated with $g$ can be performed using the method developed in [43]. Details on the computation of these proximal operators are available in [36].

\section{Initialization and Stopping Rule}

MEMM requires initial estimates $\mathbf{a}_{i}^{0}$ and $\mathbf{B}_{i}^{0}$ of the abundance vector and bundling matrix, respectively. To do so, first, MEMM estimates a multiple abundance vector $\mathbf{r}_{i}^{(0)}$ using the state-of-the-art LMM-based unmixing method [e.g., fully constrained least squares (FCLS), see line 3]. Then, an initial estimate of the single abundance vector $\mathbf{a}_{i}^{(0)}$ is computed according to (5) (see line 4). Finally, the bundling matrix $\mathbf{B}_{i}^{(0)}$ is arbitrarily initialized as the corresponding scaling factor (see line 5, where $\oslash$ stands for the element-wise division). Once initial estimates have been obtained, $\mathbf{a}_{i}^{(t+1)}$ and $\mathbf{b}^{(t+1)}$ are iteratively updated in lines $8-10$. The algorithm stops when the difference between updated and previous values of the objective function $f\left(\mathbf{B}_{i}, \mathbf{a}_{i}\right)$ is smaller than a predetermined threshold.

\section{Experiments Using Simulated Data}

First, the relevance of the proposed MEMM and its variant $\mathrm{MEMM}_{s}$ has been evaluated, thanks to experiments conducted on simulated data sets.

\section{A. Generation of Bundles}

First, $K=10$ spectra were selected from the USGS spectral library. The ten spectra were chosen so that the minimum angle between any two spectra was larger than $5^{\circ}$. This pruning prevented synthetic endmember bundles to overlap each other. Second, endmember bundles $\mathbf{E}_{k}(k=1, \ldots, K)$ were designed by randomly generated $N_{k}=30$ endmember spectra for each endmember bundle using the approach proposed in [44]. These bundles, depicted in Fig. 2, were used for generating the following two simulated data. 

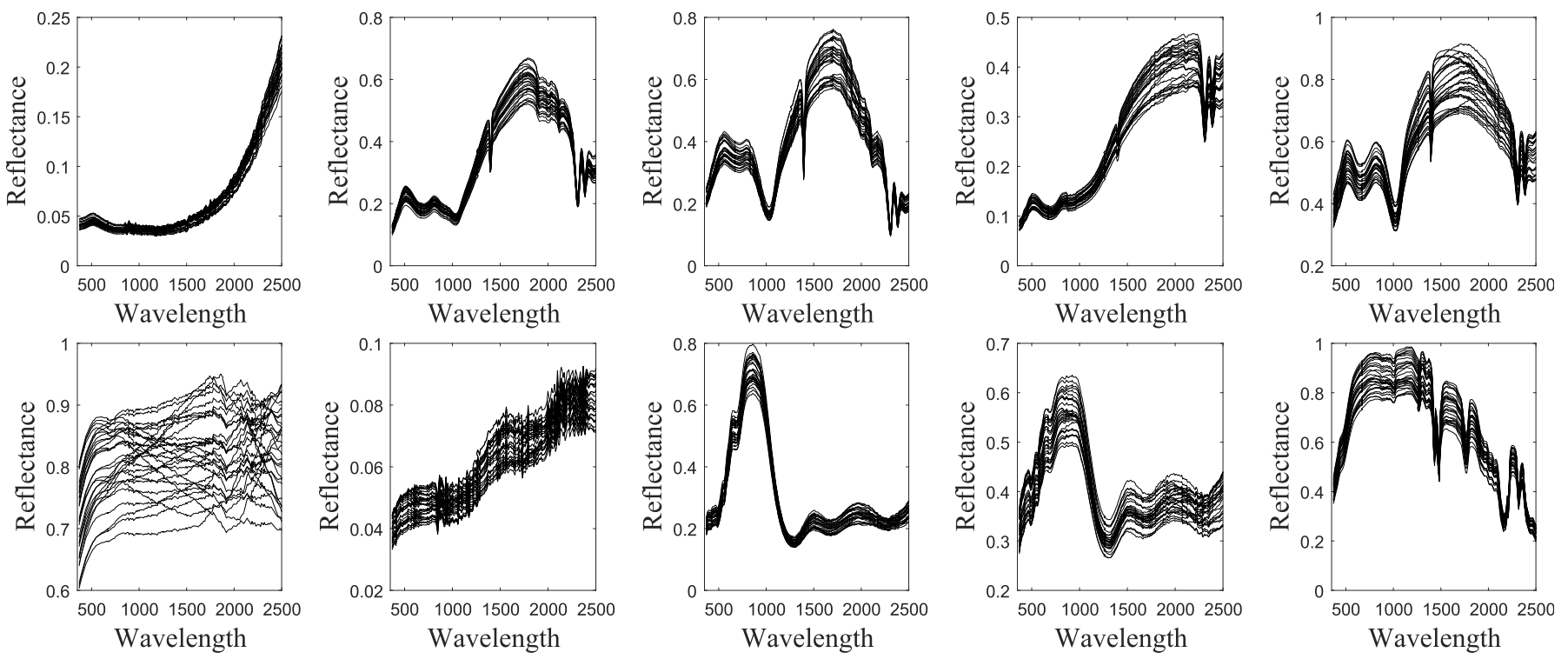

Fig. 2. Synthetically generated endmember bundles.

1) Simulated Data Set 1 (SIM1): The first data set, referred to as SIM1 in what follows, was generated using MESMA. A mixed spectrum was generated using the following five steps. First, the number of endmember classes $K$ was randomly determined in the set $(\{1, \ldots, 5\})$. Using weighted random number, a smaller number is more likely to occur in the set. Second, a random combination of endmember classes was selected. Third, one spectrum within each selected endmember class was randomly chosen. Fourth, the abundances of the selected endmember classes were randomly generated using a Dirichlet distribution to jointly ensure ANC and ASC. Finally, a mixed spectrum was generated by a linear combination of endmember spectra of the selected endmember classes and the randomly generated abundances. A set of $P=100$ mixed spectra were generated in this paper. Different amounts of additive Gaussian noise with corresponding signalto-noise ratios (SNRs) of 50, 40, and $30 \mathrm{~dB}$ were considered to the mixed spectra. A complementary scenario with lower SNR $(20 \mathrm{~dB})$ is considered in [36].

2) Simulated Data Set 2 (SIM2): SIM2 was generated using MEMM. A mixed spectrum was generated similarly as done in SIM1. The main difference is the bundling coefficients in MEMM. In order to generate the bundling coefficients, the number of spectra $N_{k}$ was randomly chosen in the set $(\{1, \ldots, 5\})$. The bundling coefficients of the randomly selected spectra were generated from a Dirichlet distribution. A mixed spectrum was generated by a linear combination of spectra, the bundling coefficients, and the abundances. As for SIM1, $P=100$ mixed spectra were generated and different amounts of Gaussian noise were also added to SIM2.

3) Simulated Data Set 3 (SIM3): For SIM3, each pixel is generated by selecting and then scaling a unique endmember spectrum belonging to a randomly chosen endmember class. More precisely, the observed pixel spectra $\mathbf{y}_{i}$ of SIM 3 can be written as $\mathbf{y}_{i}=c_{i} \mathbf{e}_{k_{i}, n_{i}}$, where $\mathbf{e}_{k_{i}, n_{i}}$ is the endmember spectrum defining the $k$ th class and $c_{i}, k_{i}$, and $n_{i}$ are randomly and uniformly drawn in the sets $(0.8,1),\{1, \ldots, K\}$, and $\left\{1, \ldots, N_{k}\right\}$, respectively. A set of $P=100$ pixels were generated, and different levels of Gaussian noise were considered. These pure pixel spectra may be mistakenly unmixed by multiple endmember classes if endmember variability affected by the scaling factor is not correctly described by the considered model. To correctly select a single endmember, strong sparsity needs to be imposed while incorporating the scaling factor.

\section{B. Compared Methods}

MEMM and $\operatorname{MEMM}_{s}$ were compared with other five methods that incorporate endmember bundles and promote sparsity: FCLS [45], sparse unmixing by variable splitting and augmented Lagrangian (SUnSAL) [46], alternating angle minimization (AAM) [47], and methods based on group lasso, elitist lasso, and fractional lasso [18], [35]. Note that, FCLS and SUnSAL can incorporate endmember bundles by considering the model in (4). Abundances estimated by SUnSAL were normalized in order to be robust to the scaling factor caused by illumination variations as discussed in [15] and [21]. FCLS and SUnSAL were chosen for comparing the proposed methods with most widely used unmixing methods that promote sparsity. AAM was selected for comparison because it is a latest variant of MESMA and it is more computationally efficient than MESMA while achieving good performance. Group lasso, elitist lasso, and fractional lasso were also included for comparison because they can incorporate the structure of endmember bundles as well as MEMM. Note that, the fractional lasso is defined by using the $\ell_{1, q}$-norm with $q=0.1$ to impose greater sparsity for the selection of endmember classes, trying to mimic the $\ell_{1,0}$-pseudo norm as discussed in [35]. MEMM, $\mathrm{MEMM}_{s}$, and the methods based on group lasso, elitist lasso, and fractional lasso require initial estimates of abundances. In order to fairly compare methods, this paper used abundances estimated by FCLS for the initial estimates required for the methods. 
SUnSAL, the methods based on group lasso, elitist lasso, and fractional lasso, and $\mathrm{MEMM}_{s}$ require a parameter $\lambda_{r}$ or $\lambda_{a}$ controlling sparsity regularization. MEMM requires two parameters $\lambda_{b}$ and $\lambda_{a}$. In order to fairly compare the methods, these parameters were empirically determined in the set $(0.0001,0.001,0.01,0.1,1,5)$ for each simulated data so that the selected values produced the highest signalto-reconstruction error $\left(\mathrm{SRE}_{a}\right)$. Parameter sensitivity of the proposed method is discussed in [36]. Finally, computation time was also discussed.

\section{Performance Criteria}

The main objective of this experiment was to assess the algorithm performance while selecting a combination of endmember classes and spectra, and estimating abundances corresponding to endmember classes and spectra. Three criteria were chosen for quantitative validation of the methods. To evaluate the quality of the reconstruction, one defines the SRE per endmember class and per endmember spectrum as [48]

$$
\begin{aligned}
\operatorname{SRE}_{a} & \equiv \mathbb{E}\left[\|\mathbf{a}\|_{2}^{2}\right] / \mathbb{E}\left[\|\mathbf{a}-\hat{\mathbf{a}}\|_{2}^{2}\right] \\
\mathrm{SRE}_{r} & \equiv \mathbb{E}\left[\|\mathbf{r}\|_{2}^{2}\right] / \mathbb{E}\left[\|\mathbf{r}-\hat{\mathbf{r}}\|_{2}^{2}\right]
\end{aligned}
$$

where $\mathbf{a}$ and $\hat{\mathbf{a}}$ are the actual and estimated abundance vectors of all pixels, and $\mathbf{r}$ and $\hat{\mathbf{r}}$ are the actual and estimated multiple abundance vectors of all pixels.

Second, the number of nonzero abundances were used to evaluate the sparsity level per endmember class and per endmember spectrum recovered by the methods (see [37], [48]). In this paper, normalized sparsity level (nSL) is defined as

$$
\begin{aligned}
\mathrm{nSL}_{a} & \equiv \frac{1}{P} \sum_{i=1}^{P} \frac{\left\|\hat{\mathbf{a}}_{i}\right\|_{0}}{\left\|\mathbf{a}_{i}\right\|_{0}} \\
\mathrm{nSL}_{r} & \equiv \frac{1}{P} \sum_{i=1}^{P} \frac{\left\|\hat{\mathbf{r}}_{i}\right\|_{0}}{\left\|\mathbf{r}_{i}\right\|_{0}} .
\end{aligned}
$$

As in [48], abundances smaller than $10^{-4}$ were considered as zero abundances. When the number of nonzero values of estimated abundances is equivalent to that of actual abundances, the nSL value becomes 1 .

Finally, to validate the performance in selecting a relevant combination of endmember classes or spectra, one defines the distance between the two actual and estimated supports (DIST) [37], [49]

$$
\begin{aligned}
\operatorname{DIST}_{a} & \equiv \frac{1}{P} \sum_{i=1}^{P} \frac{\max \left(\left|\mathcal{S}_{i}^{a}\right|,\left|\hat{\mathcal{S}}_{i}^{a}\right|\right)-\left|\mathcal{S}_{i}^{a} \cap \hat{\mathcal{S}}_{i}^{a}\right|}{\max \left(\left|\mathcal{S}_{i}^{a}\right|,\left|\hat{\mathcal{S}}_{i}^{a}\right|\right)} \\
\operatorname{DIST}_{r} & \equiv \frac{1}{P} \sum_{i=1}^{P} \frac{\max \left(\left|\mathcal{S}_{i}^{r}\right|,\left|\hat{\mathcal{S}}_{i}^{r}\right|\right)-\left|\mathcal{S}_{i}^{r} \cap \hat{\mathcal{S}}_{i}^{r}\right|}{\max \left(\left|\mathcal{S}_{i}^{r}\right|,\left|\hat{\mathcal{S}}_{i}^{r}\right|\right)}
\end{aligned}
$$

where $\mathcal{S}$ and $\hat{\mathcal{S}}$ are true and estimated support sets (i.e., indexes of nonzero values), $|\mathcal{S}|$ represents the total number of elements in the set $\mathcal{S}$, and $\cap$ stands for the intersection operator. The figures of $\mathrm{DIST}_{a}$ and $\mathrm{DIST}_{r}$ evaluated the distance between

\begin{tabular}{|c|c|c|c|c|c|c|c|c|c|}
\hline & | SNR | & FCLS & AAM & SUnSAL & Group & Elitist & Fractional & $\operatorname{MEMM}_{s}$ & MEMM \\
\hline \multirow{3}{*}{ SIM1 } & $30 \mathrm{~dB}$ & 18.0507 & 18.6585 & 19.0019 & 19.4215 & 19.2333 & 19.4756 & 14.5739 & 18.6903 \\
\hline & $40 \mathrm{~dB}$ & 22.9074 & 26.1146 & 22.9242 & 23.589 & 23.303 & 24.0219 & 14.8229 & 23.3532 \\
\hline & $50 \mathrm{~dB}$ & 27.358 & 34.4346 & 27.5203 & 27.4184 & 26.898 & 27.6176 & 14.1579 & 28.0017 \\
\hline \multirow{3}{*}{ SIM2 } & $30 \mathrm{~dB}$ & 16.1865 & 15.9426 & 16.6538 & 18.0736 & 16.4314 & 17.5376 & 12.1614 & 16.4785 \\
\hline & $40 \mathrm{~dB}$ & 21.6942 & 20.2125 & 21.6339 & 22.0263 & 21.3809 & 24.128 & 13.6682 & 22.1381 \\
\hline & $50 \mathrm{~dB}$ & 25.6617 & 22.2896 & 25.3859 & 25.9215 & 24.7702 & 26.4982 & 14.9405 & 26.5442 \\
\hline \multirow{3}{*}{ SIM3 } & $30 \mathrm{~dB}$ & 14.5914 & 14.26 & 14. & 14.3 & 14.3 & 19. & 14. & 25.6203 \\
\hline & $40 \mathrm{~dB}$ & 15.1873 & 14.6965 & 15.1079 & 14.8343 & 14.7525 & 16.9308 & 14.9971 & $\infty$ \\
\hline & $50 \mathrm{~dB}$ & 15.7588 & 14.8567 & 15.6163 & 15.204 & 15.1532 & 17.2874 & 16.4725 & $\infty$ \\
\hline
\end{tabular}
two supports of endmember classes and the distance between two supports of endmember spectra, respectively.
TABLE I

\begin{tabular}{|c|c|c|c|c|c|c|c|c|c|}
\hline & SNR & FCLS & AAM & SUnSAL & Group & Elitist & Fractional & MEMM $_{s}$ & MEMM \\
\hline \multirow{3}{*}{ SIM1 } & $30 \mathrm{~dB}$ & 1.1752 & 2.2584 & 1.7155 & 2.0398 & 1.5188 & 1.3071 & -0.457 & 0.7697 \\
\hline & $40 \mathrm{~dB}$ & 2.8611 & 5.0035 & 3.0215 & 3.0357 & 2.9957 & 2.9239 & 0.5171 & 2.7653 \\
\hline & $50 \mathrm{~dB}$ & 3.6312 & 13.9842 & 3.823 & 3.7591 & 3.7576 & 3.649 & 0.17 & 3.5721 \\
\hline \multirow{3}{*}{ SIM2 } & $30 \mathrm{~dB}$ & 1.0084 & -0.1527 & 1.4259 & 1.8935 & 1.3043 & 1.1533 & -2.0312 & 0.432 \\
\hline & $40 \mathrm{~dB}$ & 2.3323 & 1.5198 & 2.4934 & 2.5741 & 2.48 & 2.4092 & -2.1441 & 2.2462 \\
\hline & $50 \mathrm{~dB}$ & 3.2175 & $\mathbf{3 . 4 4 8 8}$ & 3.3204 & 3.3266 & 3.3666 & 3.2448 & -3.0151 & 3.2304 \\
\hline \multirow{3}{*}{ SIM3 } & $30 \mathrm{~dB}$ & 1.3047 & 0.737 & 1.4879 & 1.5672 & 1.4417 & 1.0397 & -1.8937 & 1.2851 \\
\hline & & 2.50 & 1.2622 & & 2.5154 & 2.5172 & 2.3415 & & 2.5555 \\
\hline & $50 \mathrm{~dB}$ & 3.1294 & 1.6732 & 3.215 & 3.095 & 3.1056 & 2.8264 & -1.5401 & 3.3266 \\
\hline
\end{tabular}

SRE PER ENDMEMBer Class $\left(\mathrm{SRE}_{a}\right)$. The Symbol $(\infty)$ SHOWS PERFECT RECOVERY

TABLE II

SRE PER ENDMEMBER SPECTRUM $\left(\mathrm{SRE}_{r}\right)$

\section{Results}

SRE per class was calculated for each method and is reported in Table I. For SIM1 with $50 \mathrm{~dB}$, AAM performed best among all methods. The performance of AAM, however, was degraded as SNR became lower. For data with $30 \mathrm{~dB}$, the results derived from AAM were worse than those derived from other methods except $\mathrm{MEMM}_{s}$. In addition, AAM performed poorly compared with other methods in SIM2. This showed that the MESMA-based approach (AAM) was less effective when given endmember bundles did not completely represent endmember variability present in the data and SNR of the data was low $(<40 \mathrm{~dB})$. This finding was also observed in [19]. MEMM produced better results for SIM2 with $50 \mathrm{~dB}$ than the other sparsity-methods and produced comparable results with 40 and $30 \mathrm{~dB}$. MEMM ${ }_{s}$ performed poorly, compared with all methods. In SIM3, MEMM outperformed other methods as expected. This showed that MEMM could successfully select the correct support and describe the variability caused by scaling effects while other methods failed to describe the variability. SRE per spectrum was also calculated from each method and is reported in Table II. Compared with SRE per class, SRE per spectrum was very low for all methods. This showed that the exact recovery of multiple abundances $\mathbf{r}_{i}$ was challenging under conditions where a large number of endmember spectra were present within each class.

The nSL in $\mathbf{a}_{i}$ and $\mathbf{r}_{i}$ is shown in Tables III and IV, respectively. For the number of nonzero abundances per class, the $\mathrm{nSL}_{a}$ values of MEMM were closer to 1 and performed best among all methods for SIM1, SIM2, and SIM3. This showed that the sparse constraint (the $\ell_{0}$ norm) used in MEMM successfully imposed greater sparsity and led to the selection of smaller numbers of endmember classes. For the number of nonzero abundances per spectrum, AAM and $\mathrm{MEMM}_{s}$ produced the smaller number of nonzero abundances than other methods. This was because these methods selected 
TABLE III

NSL Per Endmember Class ( $\mathrm{NSL}_{a}$ )

\begin{tabular}{c||c||cccccccc}
\hline & SNNR & FCLS & AAM & SUnSAL & Group & Elitist & Fractional & MEMM $_{s}$ & MEMM \\
\hline \multirow{3}{*}{ SIM1 1} & 30dB & 2.978 & 2.5045 & 1.8783 & 2.8432 & 4.6737 & 2.466 & 1.7205 & $\mathbf{0 . 9 8 0 5}$ \\
& 40dB & 2.8242 & 2.4623 & 2.247 & 2.6945 & 3.6512 & 2.0942 & 2.0705 & $\mathbf{1 . 1 1 4 3}$ \\
& $50 \mathrm{~dB}$ & 2.5507 & 2.303 & 1.5393 & 2.6948 & 3.0268 & 1.772 & 2.0113 & $\mathbf{1 . 0 1 8 5}$ \\
\hline \multirow{2}{*}{ SIM2 2} & 30dB $\mid$ & 2.6933 & 2.3205 & 1.7102 & 2.5335 & 3.8612 & 2.0927 & 2.1673 & $\mathbf{0 . 9 7 1 3}$ \\
& 40dB & 2.7498 & 2.2693 & 2.0768 & 2.6227 & 3.073 & 1.7193 & 2.1525 & $\mathbf{1 . 1 5 4 5}$ \\
& $50 \mathrm{~dB}$ & 2.619 & 2.1415 & 2.5373 & 2.458 & 3.0388 & 1.652 & 1.8788 & $\mathbf{1 . 0 3 6 3}$ \\
\hline \multirow{3}{*}{ SIM3 } & || $30 \mathrm{~dB}$ & 4.44 & 3.42 & 2.85 & 3.67 & 7.2 & 5.58 & 2.91 & $\mathbf{1 . 0 2}$ \\
& 40dB & 4.25 & 3.06 & 2.56 & 3.41 & 6.71 & 4.4 & 2.73 & $\mathbf{1}$ \\
& $50 \mathrm{~dB}$ & 3.9 & 2.91 & 2.55 & 5.39 & 6.53 & 4.13 & 1.92 & $\mathbf{1}$ \\
\hline
\end{tabular}

TABLE IV

NSL PER ENDMEMBER SPECTRUM (NSL $r)$

\begin{tabular}{|c|c|c|c|c|c|c|c|c|c|}
\hline & | SNR & FCLS & AAM & SUnSAL & Group & Elitist & Fractional & $\mathrm{MEMM}_{s}$ & MEMM \\
\hline \multirow{3}{*}{ SIM1 } & $30 \mathrm{~dB}$ & 6.527 & 2.5045 & 5.8797 & 22.1282 & 6.6413 & 14.545 & 1.7105 & 2.851 \\
\hline & $\begin{array}{l}40 \mathrm{~dB} \\
5 \mathrm{dBB}\end{array}$ & $\begin{array}{l}8.3438 \\
97863\end{array}$ & $\begin{array}{l}2.4623 \\
2.303\end{array}$ & $\begin{array}{l}7.0323 \\
7.6457\end{array}$ & $\begin{array}{l}15.2065 \\
1121585\end{array}$ & $\begin{array}{r}9.0737 \\
111332\end{array}$ & $\begin{array}{l}14.862 \\
162827\end{array}$ & 2.0052 & $\begin{array}{l}4.622 \\
5.4307\end{array}$ \\
\hline & & & 2.303 & 7.6457 & 12.1585 & 11.1332 & 16.2827 & 1.9468 & 5.4307 \\
\hline \multirow{3}{*}{ SIM2 } & |30dB & 4.7619 & 1.7748 & 4.5617 & 20.7952 & 5.091 & 10.6147 & 1.6059 & 1.7862 \\
\hline & 40dB & 6.2133 & 1.6638 & 5.3686 & 13.4752 & 6.9975 & 11.7411 & 1.5778 & 3.4925 \\
\hline & $50 \mathrm{~dB}$ & 7.5883 & 1.5571 & 7.3145 & 17.579 & 8.5828 & 12.8249 & 1.3488 & 5.1818 \\
\hline \multirow{3}{*}{ SIM3 } & 30dB & 10.79 & 3.42 & & & & $\begin{array}{l}43.24 \\
2 ?\end{array}$ & 65 & 2,26 \\
\hline & $40 \mathrm{~dB}$ & 12.07 & 3.06 & 11.82 & 19.94 & 14.19 & $\begin{array}{l}22.42 \\
.171\end{array}$ & 2.65 & 2.2 \\
\hline & $50 \mathrm{~dB}$ & 14.26 & 2.91 & 11.33 & 15.7 & 14.95 & 21.71 & 1.86 & 7.88 \\
\hline
\end{tabular}

TABLE V

Distance Between ACtual and Estimated Supports PER ENDMEMBER CLASS (DIST $a$ )

\begin{tabular}{|c|c|c|c|c|c|c|c|c|c|}
\hline & SNR & FCLS & AAM & SUnSAL & Group & Elitist & Fractional & $\operatorname{MEMM}_{s}$ & MEMM \\
\hline \multirow{3}{*}{ SIM1 } & $30 \mathrm{~dB}$ & 0.5893 & 0.5465 & 0.3939 & 0.5866 & 0.6967 & 0.4816 & 0.3491 & 0.1195 \\
\hline & $40 \mathrm{~dB}$ & 0.5689 & 0.5195 & 0.4616 & 0.5594 & 0.6368 & 0.3734 & 0.4498 & 0.1265 \\
\hline & $50 \mathrm{~dB}$ & 0.5137 & 0.4804 & 0.268 & 0.5479 & 0.5795 & 0.3114 & 0.418 & 0.0758 \\
\hline \multirow{3}{*}{ SIM2 } & $30 \mathrm{~dB}$ & 0.5725 & 0.5195 & 0.3775 & 0.5487 & 0.6538 & 0.4119 & 0.5025 & 0.1843 \\
\hline & $40 \mathrm{~dB}$ & 0.5789 & 0.4797 & 0.4521 & 0.5534 & 0.5952 & 0.302 & 0.4938 & 0.162 \\
\hline & $50 \mathrm{~dB}$ & 0.5345 & 0.4686 & 0.5247 & 0.5185 & 0.5825 & 0.2995 & 0.4179 & $\mathbf{0 . 1 0 3 3}$ \\
\hline \multirow{3}{*}{ SIM3 } & $30 \mathrm{~dB}$ & 0.7592 & 0.665 & 0.5783 & 0.6885 & 0.8457 & 0.7747 & 0.6 & 0.01 \\
\hline & $40 \mathrm{~dB}$ & 0.7486 & 0.6205 & 0.537 & 0.672 & 0.8237 & 0.7198 & 0.5772 & o \\
\hline & $50 \mathrm{~dB}$ & 0.7254 & 0.6253 & 0.553 & 0.7712 & 0.8149 & 0.6633 & 0.3767 & o \\
\hline
\end{tabular}

TABLE VI

Distance Between ACtual and Estimated Supports PER ENDMEMBER SPECTRUM (DIST $r$ )

\begin{tabular}{|c|c|c|c|c|c|c|c|c|c|}
\hline & SNR $\|$ & FCLS & AAM & SUnSAL & Group & Elitist & Fractional & $\operatorname{MEMM}_{s}$ & MEMM \\
\hline \multirow{3}{*}{ SIM1 } & $30 \mathrm{~dB}$ & 0.9167 & 0.7651 & 0.853 & 0.94 & 0.8963 & 0.936 & 0.7493 & 0.8115 \\
\hline & $40 \mathrm{~dB}$ & 0.8914 & 0.6721 & 0.8587 & 0.9137 & 0.8881 & 0.9215 & 0.7351 & 0.774 \\
\hline & $50 \mathrm{~dB}$ & 0.8649 & 0.555 & 0.7831 & 0.8971 & 0.8911 & 0.9294 & 0.6586 & 0.7431 \\
\hline \multirow{3}{*}{ SIM2 } & $30 \mathrm{~dB}$ & 0.9039 & 0.8389 & 0.8476 & 0.9332 & 0.8915 & 0.9094 & 0.8729 & 0.8142 \\
\hline & $40 \mathrm{~dB}$ & 0.8791 & 0.7618 & 0.8525 & 0.9167 & 0.883 & 0.9088 & 0.8175 & 0.7993 \\
\hline & $50 \mathrm{~dB}$ & 0.8738 & 0.6949 & 0.8696 & 0.9172 & 0.8816 & 0.9067 & 0.7277 & 0.8095 \\
\hline \multirow{3}{*}{ SIM3 } & $30 \mathrm{dF}$ & 0.9283 & 0.83 & 0.9049 & 0.9668 & 0.9297 & 0.9649 & 0.8 & 0.7152 \\
\hline & $40 \mathrm{~dB}$ & 0.9133 & 0.7922 & 0.8945 & 0.9377 & 0.9154 & 0.9488 & 0.785 & 0.5645 \\
\hline & $50 \mathrm{~dB}$ & 0.918 & 0.7698 & 0.8901 & 0.9222 & 0.9191 & 0.9481 & 0.5783 & 0.6955 \\
\hline
\end{tabular}

at most one spectrum within each class and enforced greater sparsity when selecting endmember spectra.

The errors in the support sets are shown in Tables V and VI. When estimating the support set per class, MEMM outperformed other methods for all data sets. Especially in SIM3 where a single class is present for each pixel, MEMM could perfectly recover the support in the data set for SNR levels of 50 and $40 \mathrm{~dB}$. This showed that the double sparsity imposed by MEMM also led to the appropriate selection of the combination of endmember classes for each pixel. The performance of MEMM, however, was degraded when estimating the support set per spectrum. Other methods also performed poorly for the support set per spectrum. This shows
TABLE VII

Computation Time For UnMIXING $P=100$ PiXels Using ENDMEMBER BundLES of $N=300$ ENDMEMBER SPECTRA

\begin{tabular}{c|cccccccc}
\hline Data & FCLS & AAM & SUNSAL & Group & Elitist & Fractional & MEMM $_{s}$ & MEMM \\
\hline SIM1 & 0.3006 & 517.7361 & $\mathbf{0 . 1 5 5}$ & 0.9517 & 1.2694 & 1.2801 & 0.4846 & 2.6625 \\
SIM2 & $\mathbf{0 . 1 9 6 6}$ & 549.1244 & 0.2616 & 0.8426 & 1.2122 & 1.2356 & 2.2311 & 2.5705 \\
SIM3 & 0.1341 & 391.1602 & $\mathbf{0 . 1 0 8 9}$ & 0.6244 & 0.8745 & 1.2769 & 1.8172 & 1.3913 \\
\hline
\end{tabular}

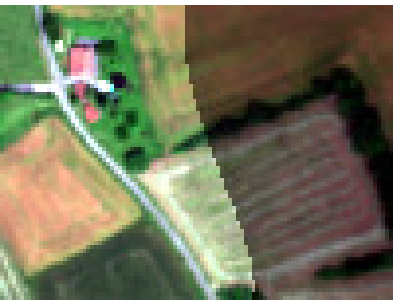

(a)

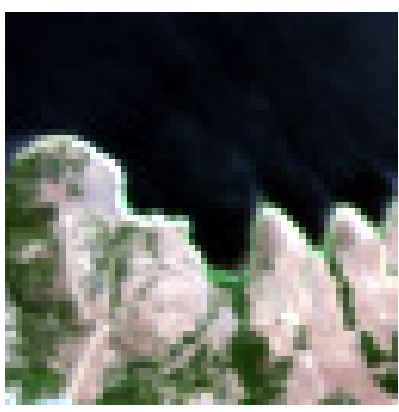

(b)
Fig. 3. Real hyperspectral images. (a) MUESLI image. (b) AVIRIS image.

that when multiple highly correlated endmember spectra are present within each class, the existing methods experience difficulty to select an optimal combination of endmember spectra.

These results also showed that better estimates in terms of extracted support (reflected by the DIST or SL values) did not necessarily lead to better SRE values. This is because even if the support and number of nonzero values are correctely recovered, estimated abundances in the correct support may be different from the actual ones. This effect may result from the auxiliary penalizations and constraints inherent to different methods (sum-to-one, nonnegativity, and various formulations of the structured sparsity).

Finally, the computation times of all methods are shown in Table VII. MEMM was more computationally expensive than FCLS, SUnSAL, group lasso, elitist lasso, and fractional lasso. The proposed methods, however, were computationally cheaper than AAM because they did not need to test a large number of combinations of endmember spectra.

\section{EXPERIMENTS Using REAL DATA}

\section{A. Description of Real Hyperspectral Images}

The methods were finally compared on two real hyperspectral images. The first hyperspectral image was acquired in June 2016, over the city of Saint-André, France, during the MUESLI airborne acquisition campaign. The image was composed of 415 spectral bands. The spectral bands affected by noise (between 1.34-1.55 and 1.80-1.98 $\mu \mathrm{m}$ ) were removed, leading to $L=345$ spectral bands. In the image scene, spatially discrete objects were present. In this paper, each spatially discrete region was assumed to be composed of a single endmember class. Endmember bundles were extracted from each region. As a consequence, large amounts of endmember variability were expected to be present within each 

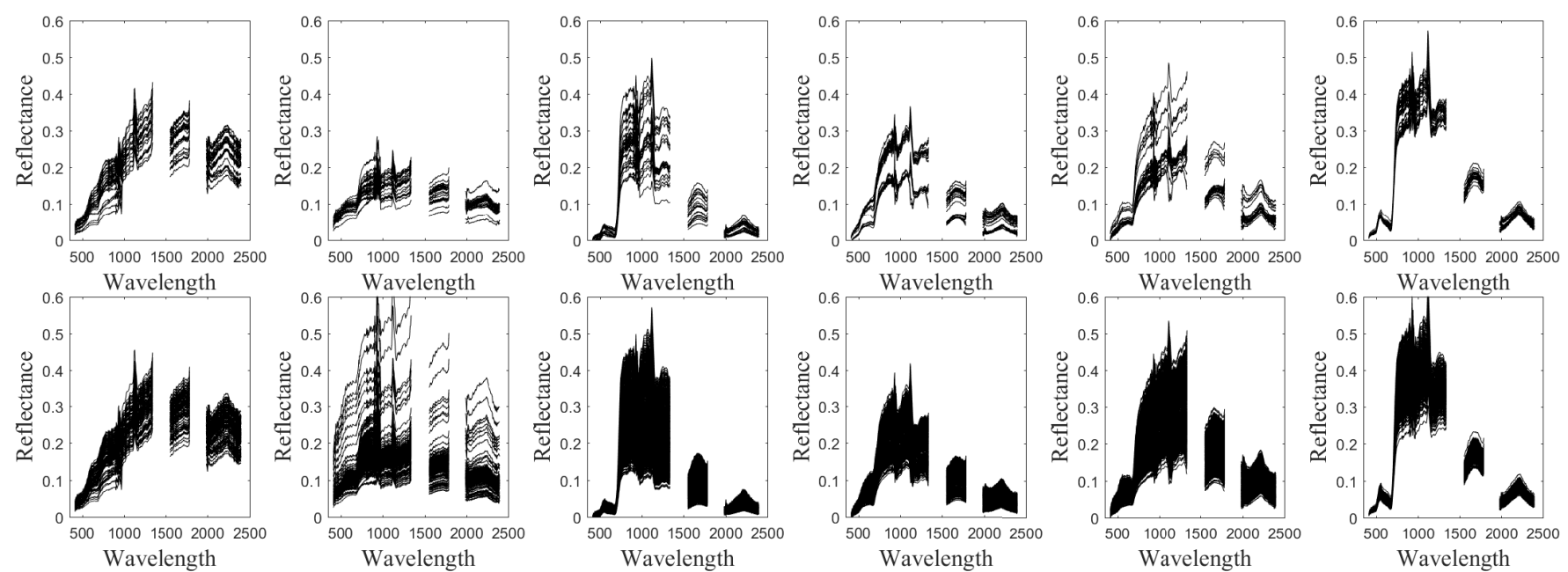

Fig. 4. (First row) Endmember bundles used for unmixing the MUESLI image. (Second row) Endmember bundles generated by MEMM.
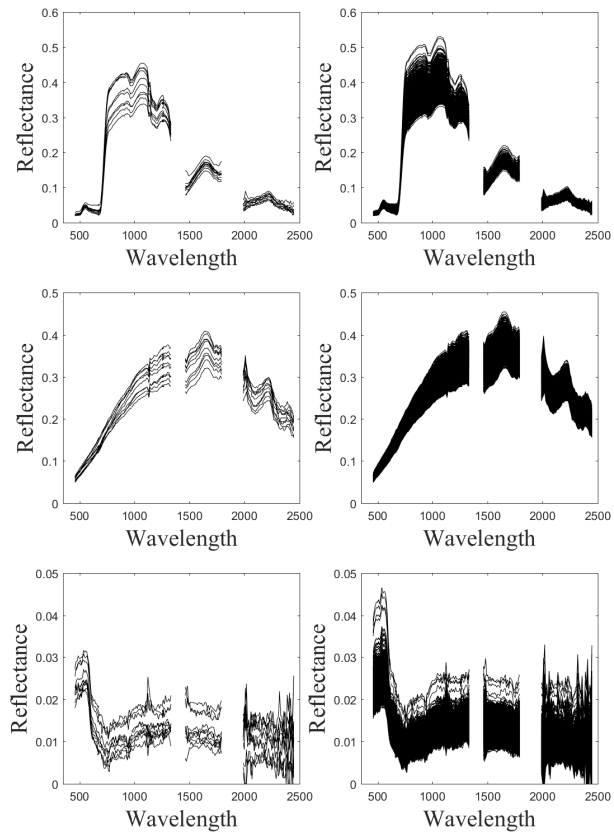

Fig. 5. (First column) Endmember bundles used for unmixing the AVIRIS image. (Second column) Endmember bundles generated by MEMM.

spatially discrete region associated with a particular class and mixed pixels were expected to be located in the boundary of these spatially discrete regions. Moreover, the scene of interest was composed of two flight lines under significantly different illumination conditions, as shown in Fig. 3(a). Thus, this image (referred to as MUESLI image) was used to evaluate whether the methods could accurately estimate abundances when large amounts of endmember variability were present. From this image, $K=6$ endmember bundles composed of a total of $N=180$ spectral signatures representing spatially discrete objects were extracted using the $n$-dimensional visualizer provided by the ENVI software. Endmember bundle extraction was done as follows. First, the $N$-dimensional visualizer was used to identify and extract pixels located near the vertices of the point clouds, as also done by state-of-theart methods [13], [27]. Multiple pixels near a particular vertex were considered as an endmember bundle. Finally, noisy pixels were removed from each endmember bundle. These bundles are represented in Fig. 4(top). Some endmember classes present in the studied area were affected by different illumination conditions. Unlike simulated data, ground truth was not available. Estimated abundances were qualitatively validated by visual inspection of the abundance maps.

The second image was acquired over Moffett Field, CA, USA, by the Airborne Visible/Infrared Imaging Spectrometer (AVIRIS). The image, depicted in Fig. 3(b), initially comprised 224 spectral bands. After the noisy spectral bands were removed, $L=178$ spectral bands remained. The area of interest, composed of a lake and a vegetated coastal area, was considered in many previous studies, e.g., to assess the performance of unmixing methods. Thus, this second image (referred to as AVIRIS image) was used to test whether the proposed method could perform at least as well as the existing methods to analyze this widely used image scene. As for the AVIRIS image, $K=3$ endmember bundles were extracted using the ENVI software and qualitative validation was conducted because of the lack of ground truth. The extracted bundles are depicted in Fig. 5(left).

\section{B. Results}

For both images, the proposed method was compared with FCLS, SUnSAL, AAM, and the methods based on group lasso, elitist lasso, and fractional lasso. The parameters $\left(\lambda_{r}, \lambda_{b}\right.$ and $\lambda_{a}$ ) required for the methods were empirically determined by qualitatively evaluating abundances derived from different values of the parameters. Finally, synthetic endmember bundles generated by MEMM were compared with endmember bundles initially used for unmixing.

Abundance maps estimated by the eight methods on the MUESLI image are depicted in Fig. 6. These maps show that the abundances estimated by MEMM and fractional lasso were more consistent at the boundary affected by different 


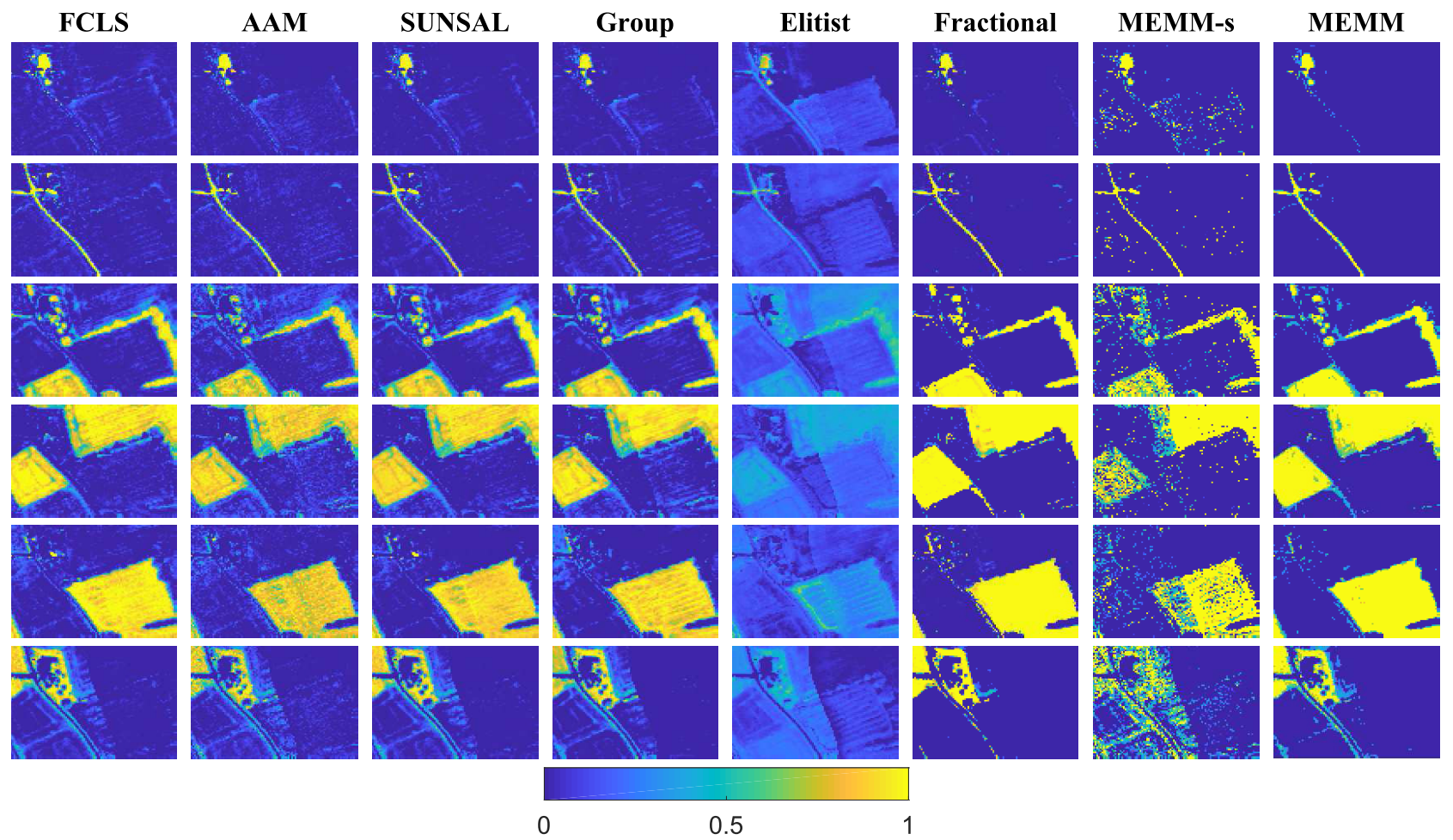

Fig. 6. MUESLI image: estimated abundance maps. (From top to bottom) Building, road, shrub, crop land 1, crop land 2, and grass.

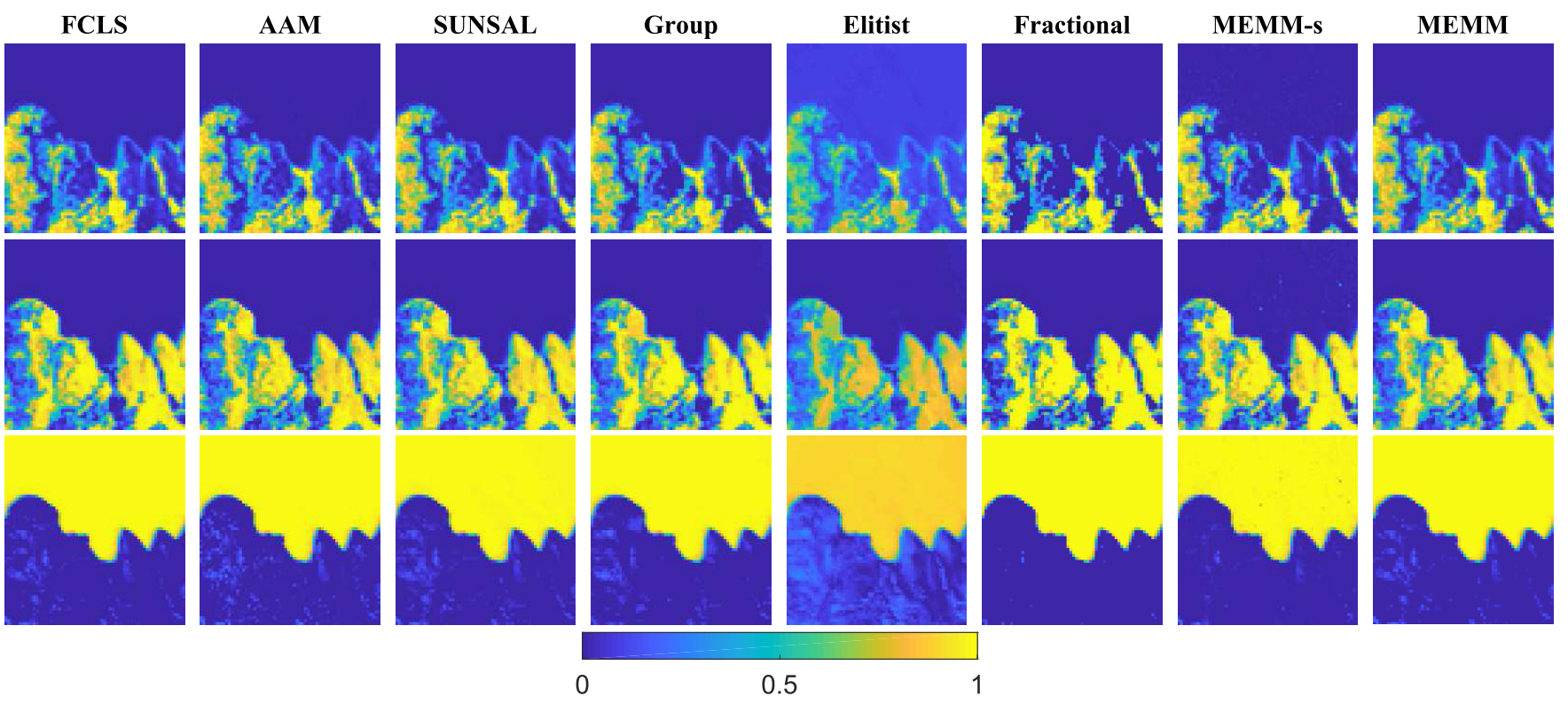

Fig. 7. AVIRIS image: abundance maps. (From top to bottom) Vegetation, soil, and water.

illumination conditions. This showed that MEMM and fractional lasso were more robust to different illumination conditions than concurrent methods. The abundances estimated by MEMM and fractional lasso were also high for each endmember class and showed less noisy. This suggested that MEMM and fractional lasso also promoted greater sparsity than other methods.

Fig. 7 shows the abundance maps estimated for the AVIRIS image. All methods except elitist lasso generated similar abundances. Abundances estimated by elitist lasso were different because it was designed to use a larger number endmember classes for unmixing each pixel. MEMM produced similar abundances when compared with FCLS, group lasso. This showed that MEMM could perform at least as well as other sparsity-based methods to unmix this well-studied test site. $\mathrm{MEMM}_{s}$, however, generated more noisy abundance maps. This showed that the initial estimates of abundances used in $\mathrm{MEMM}_{s}$ did not lead to an optimal combination of endmember spectra and the optimal abundances. Although $\mathrm{MEMM}_{s}$ may be more restrictive than MEMM, it may be important when at most one endmember spectrum from each bundle needs to be selected for each pixel. The performance 
of $\mathrm{MEMM}_{s}$ largely depends on initial estimates of multiple abundances $\mathbf{r}$. More accurate initial estimates are provided; better performance can be achieved. Therefore, MEMM $_{s}$ may achieve comparable performance in much shorter computation time than the MESMA-based methods if accurate initial abundances $\mathbf{r}$ can be estimated. This new insight is important because most existing methods focus on the development of efficient search of a combination of endmember spectra [29], [47] or the development of spectral library pruning methods to reduce computational complexity [50].

Finally, the endmember bundles recovered by MEMM were compared with endmember bundles initially used to unmix both hyperspectral images [see Figs. 4 and 5]. In Fig. 4, the synthetic endmember bundles filled the gaps that were present in the original endmember bundles. The extended endmember bundles showed more detailed spectral variability within each class in terms of both spectrum amplitudes and shapes also in Fig. 5. This enabled MEMM to generate adaptive endmember spectra within each pixel and estimate more accurate abundances even when initial endmember bundles did not completely represent endmember variability.

\section{CONCLUSION}

This paper proposed a MEMM that bridges the gap between endmember bundle-based method and data driven-based methods. MEMM appeared to be superior to the existing methods as follows: 1) it had explicit physical meaning and independently describe pixel-wise endmember spectra and abundances of each class and 2) it independently imposed double sparsity for the selection of both endmember classes and endmember spectra rather than considering structured sparsity. MEMMs were tested and compared to the state-of-the-art methods using simulated data and real hyperspectral images. MEMM showed comparable results for estimating abundances while it outperformed other methods in terms of selecting a set of endmember classes within each pixel. This paper deeply focused on sparsity constraints for both bundling coefficients and abundances. However, other constraints (e.g., spatial constraints) can be easily incorporated in the proposed unmixing framework. Future works also include the Bayesian interpretation and identifiability of the proposed model. Indeed, as most of nonconvex problems, multiple solutions may arise from this multilayer matrix factorization task in the case of overlaps between convex cones spanned by the endmember classes.

\section{ACKNOWLEDGMENT}

The authors would like to thank Prof. J. M. Bioucas-Dias, Dr. R. Heylen, Dr. L. Drumetz, and Dr. T. R. Meyer for sharing the MATLAB codes of the SUnSAL, AAM, and group/elitist/fractional lasso unmixing algorithms.

\section{REFERENCES}

[1] A. Plaza et al., "Recent advances in techniques for hyperspectral image processing," Remote Sens. Environ., vol. 113, no. 1, pp. 110-122, Sep. 2009.

[2] N. Keshava and J. F. Mustard, "Spectral unmixing," IEEE Signal Process. Mag., vol. 19, no. 1, pp. 44-57, Jan. 2002.

[3] J. M. Bioucas-Dias et al., "Hyperspectral unmixing overview: Geometrical, statistical, and sparse regression-based approaches," IEEE J. Sel. Topics Appl. Earth Observat. Remote Sens., vol. 5, no. 2, pp. 354-379, Apr. 2012.
[4] N. Dobigeon, J.-Y. Tourneret, C. Richard, J. C. M. Bermudez, S. McLaughlin, and A. O. Hero, "Nonlinear unmixing of hyperspectral images: Models and algorithms," IEEE Signal Process. Mag., vol. 31, no. 1, pp. 82-94, Jan. 2014.

[5] R. Heylen, M. Parente, and P. Gader, "A review of nonlinear hyperspectral unmixing methods," IEEE J. Sel. Topics Appl. Earth Observat. Remote Sens., vol. 7, no. 6, pp. 1844-1868, Jun. 2014.

[6] T. Uezato, M. Fauvel, and N. Dobigeon, "Hyperspectral image unmixing with LiDAR data-aided spatial regularization," IEEE Trans. Geosci. Remote Sens., vol. 56, no. 7, pp. 4098-4108, Jul. 2018.

[7] A. Zare and K. Ho, "Endmember variability in hyperspectral analysis: Addressing spectral variability during spectral unmixing," IEEE Signal Process. Mag., vol. 31, no. 1, pp. 95-104, Jan. 2014.

[8] R. J. Murphy, S. T. Monteiro, and S. Schneider, "Evaluating classification techniques for mapping vertical geology using field-based hyperspectral sensors," IEEE Trans. Geosci. Remote Sens., vol. 50, no. 8, pp. 3066-3080, Aug. 2012.

[9] T. Uezato, R. J. Murphy, A. Melkumyan, and A. Chlingaryan, "A novel endmember bundle extraction and clustering approach for capturing spectral variability within endmember classes," IEEE Trans. Geosci. Remote Sens., vol. 54, no. 11, pp. 6712-6731, Nov. 2016.

[10] B. Somers, G. P. Asner, L. Tits, and P. Coppin, "Endmember variability in spectral mixture analysis: A review," Remote Sens. Environ., vol. 115, no. 7, pp. 1603-1616, 2011.

[11] L. Drumetz, J. Chanussot, and C. Jutten, "Endmember variability in spectral unmixing: Recent advances," in Proc. IEEE Workshop Hyperspectral Image Signal Process., Evol. Remote Sens. (WHISPERS), Aug. 2016, pp. 1-4.

[12] C. A. Bateson, G. P. Asner, and C. A. Wessman, "Endmember bundles: A new approach to incorporating endmember variability into spectral mixture analysis," IEEE Trans. Geosci. Remote Sens., vol. 38, no. 2 , pp. 1083-1094, Mar. 2000.

[13] B. Somers, M. Zortea, A. Plaza, and G. Asner, "Automated extraction of image-based endmember bundles for improved spectral unmixing," IEEE J. Sel. Topics Appl. Earth Observat. Remote Sens., vol. 5, no. 2, pp. 396-408, Apr. 2012.

[14] D. A. Roberts, M. Gardner, R. Church, S. Ustin, G. Scheer, and R. O. Green, "Mapping chaparral in the Santa Monica Mountains using multiple endmember spectral mixture models," Remote Sens. Environ., vol. 65, no. 3, pp. 267-279, Sep. 1998.

[15] M. A. Veganzones et al., "A new extended linear mixing model to address spectral variability," in Proc. 6th Workshop Hyperspectral Image Signal Process., Evol. Remote Sens. (WHISPERS), Jul. 2014, pp. 1-4.

[16] M. A. Goenaga, M. C. Torres-Madronero, M. Velez-Reyes, S. J. Van Bloem, and J. D. Chinea, "Unmixing analysis of a time series of hyperion images over the Guánica dry forest in puerto rico," IEEE J. Sel. Topics Appl. Earth Observ. Remote Sens., vol. 6, no. 2, pp. 329-338, Apr. 2013.

[17] T. Uezato, R. J. Murphy, A. Melkumyan, and A. Chlingaryan, "Incorporating spatial information and endmember variability into unmixing analyses to improve abundance estimates," IEEE Trans. Image Process., vol. 25 , no. 12 , pp. 5563-5575, Dec. 2016.

[18] T. R. Meyer, L. Drumetz, J. Chanussot, A. L. Bertozzi, and C. Jutten, "Hyperspectral unmixing with material variability using social sparsity," in Proc. IEEE Int. Conf. Image Process. (ICIP), Sep. 2016, pp. 2187-2191.

[19] T. Uezato, R. J. Murphy, A. Melkumyan, and A. Chlingaryan, "A novel spectral unmixing method incorporating spectral variability within endmember classes," IEEE Trans. Geosci. Remote Sens., vol. 54, no. 5, pp. 2812-2831, May 2016.

[20] A. Halimi, N. Dobigeon, and J.-Y. Tourneret, "Unsupervised unmixing of hyperspectral images accounting for endmember variability," IEEE Trans. Image Process., vol. 24, no. 12, pp. 4904-4917, Dec. 2015.

[21] L. Drumetz, M.-A. Veganzones, S. Henrot, R. Phlypo, J. Chanussot, and C. Jutten, "Blind hyperspectral unmixing using an extended linear mixing model to address spectral variability," IEEE Trans. Image Process., vol. 25, no. 8, pp. 3890-3905, Aug. 2016.

[22] P.-A. Thouvenin, N. Dobigeon, and J.-Y. Tourneret, "Hyperspectral unmixing with spectral variability using a perturbed linear mixing model," IEEE Trans. Signal Process., vol. 64, no. 2, pp. 525-538, Jan. 2016

[23] D. Hong, N. Yokoya, J. Chanussot, and X. X. Zhu, "Learning a low-coherence dictionary to address spectral variability for hyperspectral unmixing," in Proc. IEEE Int. Conf. Image Process. (ICIP), Sep. 2017, pp. 235-239. 
[24] Y. Zhou, A. Rangarajan, and P. D. Gader, "A Gaussian mixture model representation of endmember variability in hyperspectral unmixing," IEEE Trans. Image Process., vol. 27, no. 5, pp. 2242-2256, May 2018.

[25] A. Zare, P. Gader, and G. Casella, "Sampling piecewise convex unmixing and endmember extraction," IEEE Trans. Geosci. Remote Sens., vol. 51, no. 3, pp. 1655-1665, Mar. 2013.

[26] R. Rubinstein, M. Zibulevsky, and M. Elad, "Double sparsity: Learning sparse dictionaries for sparse signal approximation," IEEE Trans. Signal Process., vol. 58, no. 3, pp. 1553-1564, Mar. 2010.

[27] M. Xu, L. Zhang, and B. Du, "An image-based endmember bundle extraction algorithm using both spatial and spectral information," IEEE J. Sel. Topics Appl. Earth Observ. Remote Sens., vol. 8, no. 6, pp. 2607-2617, Jun. 2015.

[28] P. E. Dennison and D. A. Roberts, "Endmember selection for multiple endmember spectral mixture analysis using endmember average RMSE," Remote Sens. Environ., vol. 87, nos. 2-3, pp. 123-135, 2003.

[29] L. Tits, B. Somers, R. Heylen, P. Scheunders, and P. Coppin, "Improving the efficiency of MESMA through geometric unmixing principles," Proc. SPIE, vol. 8892, p. 88920Q, Oct. 2013.

[30] L. Demarchi, F. Canters, J. C.-W. Chan, and T. Van de Voorde, "Multiple endmember unmixing of CHRIS/proba imagery for mapping impervious surfaces in urban and suburban environments," IEEE Trans. Geosci. Remote Sens., vol. 50, no. 9, pp. 3409-3424, Sep. 2012.

[31] J. Sigurdsson, M. O. Ulfarsson, and J. R. Sveinsson, "Hyperspectral unmixing with $l_{q}$ regularization," IEEE Trans. Geosci. Remote Sens., vol. 52, no. 11, pp. 6793-6806, Nov. 2014.

[32] C. Y. Zheng, H. Li, Q. Wang, and C. L. P. Chen, "Reweighted sparse regression for hyperspectral unmixing," IEEE Trans. Geosci. Remote Sens., vol. 54, no. 1, pp. 479-488, Jan. 2016.

[33] W. He, H. Zhang, and L. Zhang, "Total variation regularized reweighted sparse nonnegative matrix factorization for hyperspectral unmixing," IEEE Trans. Geosci. Remote Sens., vol. 55, no. 7, pp. 3909-3921, Jul. 2017.

[34] M.-D. Iordache, J. M. Bioucas-Dias, and A. Plaza, "Hyperspectral unmixingwith sparse group lasso," in Proc. IEEE Int. Conf. Geosci. Remote Sens. (IGARSS), Jul. 2011, pp. 3586-3589.

[35] L. Drumetz, T. R. Meyer, J. Chanussot, A. L. Bertozzi, and C. Jutten. (2018). "Hyperspectral image unmixing with endmember bundles and group sparsity inducing mixed norms." [Online]. Available: https://arxiv.org/abs/1805.10232

[36] T. Uezato, M. Fauvel, and N. Dobigeon, "Hyperspectral unmixing with spectral variability using adaptive bundles and double sparsityComplementary results," Univ. Toulouse, Toulouse, France, Tech. Rep., 2018.

[37] F. Chen, K. Wang, and T. F. Tang, "Spectral unmixing using a sparse multiple-endmember spectral mixture model," IEEE Trans. Geosci. Remote Sens., vol. 54, no. 10, pp. 5846-5861, Oct. 2016.

[38] T. Imbiriba, R. A. Borsoi, and J. C. M. Bermudez. (2017). "Generalized linear mixing model accounting for endmember variability." [Online]. Available: https://arxiv.org/abs/1710.07723

[39] R. Rajabi and H. Ghassemian, "Spectral unmixing of hyperspectral imagery using multilayer NMF," IEEE Geosci. Remote Sens. Lett., vol. 12, no. 1, pp. 38-42, Jan. 2015.

[40] N. Akhtar and A. Mian, "RCMF: Robust constrained matrix factorization for hyperspectral unmixing," IEEE Trans. Geosci. Remote Sens., vol. 55, no. 6, pp. 3354-3366, Jun. 2017

[41] C. Zhao, G. Zhao, and X. Jia, "Hyperspectral image unmixing based on fast kernel archetypal analysis," IEEE J. Sel. Topics Appl. Earth Observ. Remote Sens., vol. 10, no. 1, pp. 331-346, Jan. 2017.

[42] J. Bolte, S. Sabach, and M. Teboulle, "Proximal alternating linearized minimization for nonconvex and nonsmooth problems," Math. Program. vol. 146, nos. 1-2, pp. 459-494, 2014.

[43] K. Anastasios, S. Becker, V. Cevher, and C. Koch, "Sparse projections onto the simplex," in Proc. Int. Conf. Mach. Learn., 2013, pp. 235-243.

[44] P.-A. Thouvenin, N. Dobigeon, and J.-Y. Tourneret, "Online unmixing of multitemporal hyperspectral images accounting for spectral variability," IEEE Trans. Image Process., vol. 25, no. 9, pp. 3979-3990, Sep. 2016.

[45] J. M. Bioucas-Dias and M. A. T. Figueiredo, "Alternating direction algorithms for constrained sparse regression: Application to hyperspectral unmixing," in Proc. 2nd Workshop Hyperspectral Image Signal Process., Evol. Remote Sens. (WHISPERS), Jun. 2010, pp. 1-4.

[46] M.-D. Iordache, J. Bioucas-Dias, and A. Plaza, "Sparse unmixing of hyperspectral data," IEEE Trans. Geosci. Remote Sens., vol. 49, no. 6, pp. 2014-2039, Jun. 2011.
[47] R. Heylen, A. Zare, P. Gader, and P. Scheunders, "Hyperspectral unmixing with endmember variability via alternating angle minimization," IEEE Trans. Geosci. Remote Sens., vol. 54, no. 8, pp. 4983-4993, Aug. 2016.

[48] M.-D. Iordache, J. M. Bioucas-Dias, and A. Plaza, "Collaborative sparse regression for hyperspectral unmixing," IEEE Trans. Geosci. Remote Sens., vol. 52, no. 1, pp. 341-354, Jan. 2014.

[49] M. Elad, Sparse and Redundant Representations: From Theory to Applications in Signal and Image Processing. Springer, 2010.

[50] M.-D. Iordache, L. Tits, J. M. Bioucas-Dias, A. Plaza, and B. Somers, "A dynamic unmixing framework for plant production system monitoring," IEEE J. Sel. Topics Appl. Earth Observat. Remote Sens., vol. 7, no. 6, pp. 2016-2034, Jun. 2014.

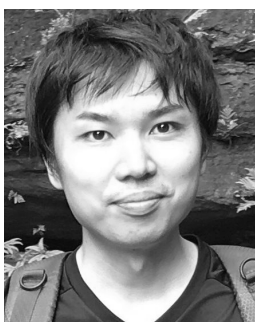

Tatsumi Uezato received the M.Sc. degree in science from Nagoya University, Nagoya, Japan, in 2012, and the Ph.D. degree from The University of Sydney, Sydney, NSW, Australia, in 2016, with a focus on the use of machine learning for estimating abundances of materials from hyperspectral imagery with specific reference to incorporating spectral and spatial information.

He is currently a Post-Doctoral Researcher with the Institut de Recherche en Informatique de Toulouse, Toulouse, France, where he has developed spectral unmixing methods using hyperspectral and LiDAR data. His research interests include hyperspectral image processing, remote sensing, and machine learning.

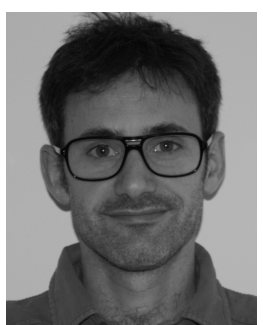

Mathieu Fauvel (SM'16) received the Degree in electrical engineering and the M.S. and Ph.D. degrees in image and signal processing from the Grenoble Institute of Technology (Grenoble INP), Grenoble, France, in 2004, 2004, and 2007, respectively.

In 2007, he joined Grenoble INP as a Teaching Assistant. From 2008 to 2010, he was a PostDoctoral Research Associate with the MISTIS Team, National Institute for Research in Computer Science and Control (INRIA), Grenoble. From 2011 to 2018, he was an Associate Professor with the National Polytechnic Institute of Toulouse (ENSAT-University of Toulouse) within the DYNAFOR Lab (University of Toulouse-INRA), Toulouse, France. From 2013 to 2016, he was the President of the French Chapter of the Geoscience and Remote Sensing Society (GRSS). He is currently in charge of the European GRSS Chapters activities. Since 2018, he has been a Full Researcher with CESBIO-INRA, Toulouse. His research interests include remote sensing, data fusion, pattern recognition, multicomponent signal, and image processing.

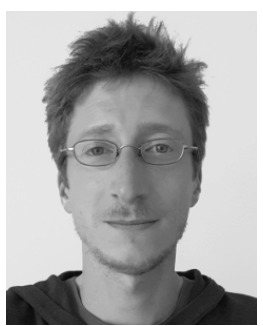

Nicolas Dobigeon (S'05-M'08-SM'13) was born in Angoulême, France, in 1981. He received the Engineering degree in electrical engineering from ENSEEIHT, Toulouse, France, in 2004, and the M.Sc. and Ph.D. degrees in signal processing from Toulouse INP, Toulouse, in 2004 and 2007, respectively.

From 2007 to 2008, he was a Post-Doctora Research Associate with the Department of Electrical Engineering and Computer Science, University of Michigan, Ann Arbor, MI, USA. Since 2008, he has been with Toulouse INP, University of Toulouse, Toulouse, where he is currently a Professor. He conducts his research activities within the Signal and Communications Group, Institut de Recherche en Informatique de Toulouse Laboratory, Toulouse, and he is also an Affiliated Faculty Member of the Telecommunications for Space and Aeronautics Cooperative Laboratory, Toulouse. Since 2017, he has been a Junior Member of the Institut Universitaire de France, France. His research interests include statistical signal and image processing, with a particular interest in Bayesian inverse problems with applications to remote sensing, biomedical imaging, and microscopy. 